\title{
Uniqueness of $\mathcal{N}=2$ and 3 pure supergravities in 4D
}

\author{
Nicolas Boulanger, ${ }^{a, 1}$ Bernard Julia $^{b}$ and Lucas Traina ${ }^{a, 2}$ \\ ${ }^{a}$ Group of Mechanics and Gravitation, Physique théorique et mathématique, \\ University of Mons - UMONS, \\ 20 Place du Parc, 7000 Mons, Belgium \\ ${ }^{b} L P T-E N S, P S L$ and $C N R S$, \\ 24 rue Lhomond, 75231 Paris CEDEX, France \\ E-mail: nicolas.boulanger@umons.ac.be, bjulia@lpt.ens.fr, \\ lucas.traina@umons.ac.be
}

ABSTRACT: After proving the impossibility of consistent non-minimal coupling of a real Rarita-Schwinger gauge field to electromagnetism, we re-derive the necessity of introducing the graviton in order to couple a complex Rarita-Schwinger gauge field to electromagnetism, with or without a cosmological term, thereby obtaining $\mathcal{N}=2$ pure supergravity as the only possibility. These results are obtained with the BRST-BV deformation method around the flat and (A)dS backgrounds in 4 dimensions. The same method applied to $n_{v}$ vectors, $\mathcal{N}$ real spin-3/2 gauge fields and at most one real spinor field also requires gravity and yields $\mathcal{N}=3$ pure supergravity as well as $\mathcal{N}=1$ pure supergravity coupled to a vector supermultiplet, with or without cosmological terms. Independently of the matter content, we finally derive strong necessary quadratic constraints on the possible gaugings for an arbitrary number of spin-1 and spin-3/2 gauge fields, that are relevant for larger supergravities.

KeYwORDS: Extended Supersymmetry, Gauge Symmetry, Supergravity Models

ARXIV EPRINT: 1802.02966

\footnotetext{
${ }^{1}$ Senior Research Associate of the F.R.S.-FNRS (Belgium).

${ }^{2}$ Research Fellow of the F.R.S.-FNRS (Belgium).
} 


\section{Contents}

1 Introduction 1

2 Cohomological reformulation of the deformation problem 5

2.1 Cohomological approach 5

2.2 First starting point: free BV functionals in flat background 7

2.3 Second starting point: anti de Sitter background 9

3 Uniqueness of $\mathcal{N}_{4}=2$ and $\mathcal{N}_{4}=3$ pure supergravities 10

3.1 Uniqueness of $\mathcal{N}=2$ pure sugra 11

$\begin{array}{ll}3.2 & \text { Obstruction to the U(1) minimal coupling } \\ & 17\end{array}$

$\begin{array}{lll}3.3 & \text { Deformations around AdS background } & 18\end{array}$

3.4 General and specific quadratic constraints 22

4 Conclusions and perspectives $\quad 29$

$\begin{array}{ll}\text { A Conventions and some Diracology } & 30\end{array}$

B Appearance of conserved currents in gaugings $\quad 31$

\section{Introduction}

The problem of consistent couplings between a complex massless spin- $\frac{3}{2}$ field and electromagnetism in $4 \mathrm{D}$ has been studied long ago $[1,2]$. It was re-investigated very recently $[3,4]$ by Adler who argued that the gauge invariance of the massless Rarita-Schwinger ${ }^{1}$ field could harmlessly be lost in the minimal coupling to electromagnetism. However, in [6] it was rigorously shown that for the model $[3,4]$ gauge invariance is crucial. More generally, it appears that gauge invariance can only be deformed and should never be reduced or augmented in the process of introducing perturbative interactions among a given set of gauge fields. Keeping gauge invariance of constant size, which one calls consistency, is our main assumption, together with locality, no more than two derivatives in the Lagrangian and perturbative deformations. Slightly more precisely, we require that the number of gauge parameters is preserved, although the form of the gauge transformation laws can be deformed perturbatively.

In this paper we start a systematic investigation, in four spacetime dimensions, of all the possible consistent couplings of a set of $\mathcal{N}_{4} \geqslant 1$ massless spin-3/2 fields and a set of $n_{v}$

\footnotetext{
${ }^{1}$ In this paper, by "massless Rarita-Schwinger field" we actually mean a spin- $\frac{3}{2}$ gauge field in the Majorana representation. In the original paper by Rarita and Schwinger [5], the spinor, whether massive or massless, is in the Dirac representation. Throughout this paper, a spinor in the Majorana representation will be called real, although we do not specify the representation used for the Dirac matrices.
} 
vector gauge fields. We also allow, if necessary, for the introduction of at most one graviton and at most one real spin- $\frac{1}{2}$ field. On the technical side, we follow the cohomological approach of [7] based on the antifield $[8,9]$ reformulation of the perturbative deformation procedure exposed in [10]. We refer to [11] where this framework was applied to deform a sum of Fierz-Pauli actions and a pedagogical exposition was given on the general procedure. See e.g. [12-15] for recent works where this approach has been followed. At first order in the infinitesimal deformation parameters one typically finds linear constraints restricting the number of possible couplings. At second order in the parameters one obtains quadratic constraints among them.

In this note, among other things we re-derive and strengthen the old result on the impossibility of consistent coupling (minimal or non minimal) of one real Rarita-Schwinger field to electromagnetism. Then, starting with two real Rarita-Schwinger gauge fields coupled to Maxwell fields, we see that the introduction of the graviton is necessary in order to ensure consistency at second order in the infinitesimal deformation parameters. Starting from three Rarita-Schwinger gauge fields coupled to vector gauge fields, this time it is the introduction of both the graviton and one Majorana spin- $\frac{1}{2}$ field that is necessary in order to ensure the consistency of the coupling at second order. As a matter of fact, in these two situations the coupling constant $\kappa$ gets related to Newton's constant $G_{N}=\frac{\kappa^{2}}{32 \pi}$, where we have set $c=1$. Thereby, the consistency of the couplings of massless spin- $\frac{3}{2}$ fields to vector gauge fields naturally leads to the uniqueness of $\mathcal{N}_{4}=2$ and $\mathcal{N}_{4}=3$ pure supergravities in 4 dimensions, with and without cosmological constant terms. Restricting the matter spectrum to contain at most one spin- $\frac{1}{2}$ field and no scalars excludes the theories of supergravity with $\mathcal{N}_{4} \geqslant 4$ and excludes the matter-coupled supergravities, except for $\mathcal{N}_{4}=1$ supergravity coupled to one vector $(1,1 / 2)$ multiplet [16].

Still, independently of the restriction of the scalar and spin- $\frac{1}{2}$ field contents, we derive strong necessary constraints on the possible gaugings for an arbitrary number of massless spin- $\frac{3}{2}$ and spin- 1 fields. We defer the analysis of couplings with more than one Majorana spin- $\frac{1}{2}$ field and some scalar fields to another paper. It is anticipated that the introduction of several Majorana spinor fields together with some scalar fields will be necessary to ensure consistency of pure $\mathcal{N}_{4} \geqslant 4$ supergravity and of matter-coupled supergravities. An appealing long term goal would be to automatize the computation of the relevant cohomologies for the construction and classification of solutions.

We exploit the exhaustivity of the cohomological reformulation [7] of the consistent coupling problem in the antifield formalism of Batalin and Vilkovisky [8, 9] (BV, for short) in order to classify all the possible couplings involving a set of $\mathcal{N}_{4}$ spin- $\frac{3}{2}$ gauge fields, $n_{v}$ Maxwell vectors, a single massless spin-2 field - if necessary - together with matter in the form of at most one Majorana spinor. Some of our results can be summarised in the form of a theorem and a corollary.

Theorem (Uniqueness of $\mathcal{N}_{4}=2$ and $\mathcal{N}_{4}=3$ supergravities). With the assumptions of locality, Poincaré invariance, parity invariance, no more than two derivatives in the deformed Lagrangian and at most one Majorana spin- $\frac{1}{2}$ "matter" field, the $\mathcal{N}_{4}=2$ and $\mathcal{N}_{4}=3$ supergravity theories of $[17,18]$ are the unique ways to consistently couple at least two real 
spin- $\frac{3}{2}$ gauge fields to vector gauge fields around $4 D$ Minkowski spacetime. In particular, the cosmological constant terms appear as consistent deformations. Neither local supersymmetry, nor general covariance nor even minimal coupling are assumed: they all appear as a result of the consistency of the deformation. In particular, dynamical gravity and its diffeomorphism invariance are necessarily required, thereby leading to background independence.

The zero cosmological constant limit of the $\mathcal{N}_{4}=2$ and $\mathcal{N}_{4}=3 A d S_{4}$ supergravity theories of $[17,18]$ is smooth and gives rise to the two earlier models [19] and [20]. A priori, there could have been other theories coupling two and three Rarita-Schwinger gauge fields to electromagnetism and to $\mathrm{SO}(3)$ Yang-Mills gauge fields. In fact, there are no others and this follows from our theorem. A corollary can be stated as follows:

Corollary. The only consistent way to couple the $\mathcal{N}_{4}=1$ massless multiplet $\left(1, \frac{3}{2}\right)$ to the $\mathcal{N}_{4}=1$ gravitational multiplet $\left(\frac{3}{2}, 2\right)$ is through $\mathcal{N}_{4}=2$ supergravity. Analogously, the only consistent way to couple the $\mathcal{N}_{4}=2$ massless multiplet $\left(\frac{1}{2}, 1,1, \frac{3}{2}\right)$ to the $\mathcal{N}_{4}=2$ gravitational multiplet $\left(1, \frac{3}{2}, \frac{3}{2}, 2\right)$ is through $\mathcal{N}_{4}=3$ supergravity.

In particular, our results reproduce the well-known impossibility to minimally couple some massless spin- $\frac{3}{2}$ fields to electromagnetism around flat Minkowski spacetime. As was found long ago in $[17,18]$, the solution to this problem requires the introduction of a (negative) cosmological constant as well as dynamical gravity. As stated in our theorem, the theories with a nonvanishing cosmological constant are obtained from theories without any cosmological constant, starting from flat spacetime, where infinitesimal cosmological constant terms appear as deformations that can be continued to all orders. As was shown in [11] in the case of massless spin-2 fields around Minkowski spacetime, an infinitesimal cosmological constant term appears as a consistent, first-order deformation that can be continued to all orders, without introducing any explicit Cartesian coordinate $x^{\mu}$ dependence at any stage of the deformation process, leading to the Einstein-Hilbert Lagrangian with finite cosmological constant term. In this sense, our theorem stated above is the natural generalisation of the theorems proved in [11] and in [21].

In the present paper, however, our strategy is slightly different, although it leads to the same end result stated in our above theorem: in the presence of massless spin- $\frac{3}{2}$ fields and waiving the requirement of Poincaré invariance (in particular, allowing for explicit $x^{\mu}$ dependence in the deformed Lagrangian), the infinitesimal cosmological-constant deformation of the quadratic Lagrangian around flat spacetime can be continued to all orders to give the quadratic action consisting of the sum of the Fierz-Pauli action in $A d S_{4}$ with cosmological constant term and several Rarita-Schwinger actions with mass-like terms around $A d S_{4}$. That the cosmological constant must be negative was re-derived in [21], consistently with the original finding of [22]. Then, from this quadratic Lagrangian in $A d S_{4}$ background, we pursue the process of consistent deformation, this time looking for all the possible $\mathrm{SO}(2,3)$-invariant cubic vertices around $A d S_{4}$. The advantage of this strategy is that the cosmological constant terms are finite from the very beginning and the minimal coupling terms arise at the first order in deformation, instead of appearing at second order as it is the case when one sticks to Minkowski background and Poincaré invariance at every 
stage. The end result is the same in both ways: gravity is required for consistency of the deformation, and with it, diffeomorphism invariance that washes away the relevance of the initial background.

We actually obtained other results that are not contained in our theorem: (A) Whenever the Majorana spin- $\frac{1}{2}$ field is required for the coupling of massless spin- $\frac{3}{2}$ fields to vector gauge fields, the spin- $\frac{1}{2}$ field must be massless for consistency. (B) If one relaxes the assumption that there should be at least two real spin- $\frac{3}{2}$ gauge fields in the spectrum, we found that altogether three interacting models can arise for the coupling of the Maxwell fields to the spin- $\frac{3}{2}$ gauge fields:

(B.1) One of the $n_{v}$ vectors is coupled to one spin- $\frac{3}{2}$ gauge fields, the spin- $\frac{1}{2}$ field and gravity according to the theory [16]. This model describes the coupling of a (gauged) $\mathcal{N}_{4}=1$ supermultiplet $(2,3 / 2)$ to a (rigid) $\mathcal{N}_{4}=1$ vector supermultiplet $(1,1 / 2)$. The other spin- $\frac{3}{2}$ gauge fields remain decoupled from all the rest. We found that the model in [16] can be extended so as to include the cosmological constant terms, as in [22] or in the $\mathrm{SO}(3)$ model of $[17] ;^{2}$

(B.2) Only one of the $n_{v}$ vectors, that is identified with the factor $\mathrm{U}(1)$ of electromagnetism, is coupled to two of the spin- $\frac{3}{2}$ gauge fields through pure $\mathcal{N}_{4}=2$ supergravity, with and without cosmological constant terms $[17,19]$, the other $n_{v}-1$ vectors remaining decoupled from the remaining $\mathcal{N}_{4}-2$ spin- $\frac{3}{2}$ gauge fields;

(B.3) Only three of the $n_{v}$ vectors, that will be identified with an $\mathrm{SO}(3)$-valued Yang-Mills connection, are coupled to three of the Rarita-Schwinger gauge fields, minimally and non-minimally, with and without cosmological constant terms [17, 20].

Finally, (C) Strong necessary constraints, valid regardless of the number of vectors and Rarita-Schwinger gauge fields, are found on the possible gaugings; they are independent of the matter content of the spectrum.

The plan of the paper is as follows. In the next section 2 , we very briefly review the cohomological framework [7] for introducing consistent interactions in a gauge theory. In section 3, we apply these techniques and the methodology of $[23,24]$ in order to investigate the possible couplings between a set of real Rarita-Schwinger gauge fields and several vector gauge fields, possibly with gravity and a real spin- $\frac{1}{2}$ field. Section 3 leads, as main result, to the uniqueness of $\mathcal{N}_{4}=2$ and $\mathcal{N}_{4}=3$ pure supergravities, with and without cosmological constant terms. In subsection 3.4 we also derive general quadratic constraints on possible gaugings. These are necessary constraints that must be satisfied independently of the matter content of the undeformed Lagrangian and are therefore also valid for extended supergravities with $\mathcal{N}_{4} \geqslant 4$. Section 4 gives some outlooks and conclusions. In a first appendix we gather some identities on spinors and Clifford algebra, while the second appendix comments the results of the paper in terms of the original Noether procedure for introducing interactions from gaugings of rigid (non-abelian) symmetries. The global

\footnotetext{
${ }^{2}$ We could not find any reference mentioning this extension of the model [16], although it must be known to experts.
} 
symmetries being "gauged" disappear as such in the deformation: they are subsumed in the emerging non-abelian gauge group. The BRST-BV approach followed in the body of the text is different, as it starts from abelian gauge symmetries that are then consistently deformed to result in non-abelian gauge theories.

\section{Cohomological reformulation of the deformation problem}

In this section, we briefly review the cohomological procedure [7] for perturbative deformation of a Lagrangian gauge theory, exemplifying it on the free theories describing massless spin- $s$ fields around flat and $A d S_{4}$ backgrounds for $s \in\left\{\frac{1}{2}, 1, \frac{3}{2}, 2\right\}$. We also present our conventions and notation for spinors.

\subsection{Cohomological approach}

Initial theory. In order to introduce consistent interactions to an initial theory characterised by an action $S_{0}\left[\varphi^{i}\right]$ invariant under the gauge transformations ${ }^{3} \Delta_{0} \varphi^{i}=R_{0}{ }^{i}{ }_{\alpha} \epsilon^{\alpha}$, an appropriate framework is provided by the reformulation [7] of the Noether deformation procedure in the Batalin-Vilkovisky (BV) antifield formalism [8, 9]. An advantage of the antifield formalism is that the whole gauge structure, including initial action $S_{0}\left[\varphi^{i}\right]=\int d^{4} x \mathcal{L}_{0}$, its gauge invariances and the gauge algebra, is captured by the BV master action (or BV functional) $W_{0}$, see e.g. $[25,26]$ for reviews. The master action is a functional

$$
W_{0}\left[\varphi^{i}, C^{\alpha}, \varphi_{i}^{\star}, C_{\alpha}^{\star}\right]=S_{0}\left[\varphi^{i}\right]+\varphi_{i}^{\star} R_{0}{ }^{i}{ }_{\alpha} C^{\alpha}+\frac{1}{2} C_{\alpha}^{\star} f_{0}{ }^{\alpha}{ }_{\beta \gamma} C^{\beta} C^{\gamma}+\ldots,
$$

that satisfies the classical master equation

$$
\left(W_{0}, W_{0}\right)=0
$$

In this equation, the BV antibracket is the graded-odd Lie bracket defined by

$$
(X, Y):=\int d^{4} x\left[\frac{\delta^{R} X}{\delta \Phi^{A}(x)} \frac{\delta^{L} Y}{\delta \Phi_{A}^{\star}(x)}-\frac{\delta^{R} X}{\delta \Phi_{A}^{\star}(x)} \frac{\delta^{L} Y}{\delta \Phi^{A}(x)}\right],
$$

on the extended space locally coordinatised by $\Phi^{A}:=\left(\varphi^{i}, C^{\alpha}\right)$, the original fields and ghosts for the type of theories we will be interested in, and their canonically conjugated antifields $\Phi_{A}^{\star}=\left(\varphi_{i}^{\star}, C_{\alpha}^{\star}\right)$. The Lagrangian, gauge variations of the fields and structure functions of the gauge algebra are contained in the first, second and third term of the master action (2.1), respectively. The BRST differential $s$ associated with the initial theory, acts as

$$
s \bullet:=\left(W_{0}, \bullet\right) .
$$

That it is a differential follows from the master equation (2.2). A $\mathbb{Z}$ grading is associated with $s$, that is called the ghost number $g h$. The BRST differential increases the ghost number by one unit. For the type of theories we are interested in, $s$ decomposes into the sum of two differentials: $s=\delta+\gamma$. For a free theory action $S_{0}$, the Koszul-Tate differential

\footnotetext{
${ }^{3}$ We use De Witt's condensed notation.
} 
$\delta$ acts only on the antifields $\Phi_{A}^{\star}$, while $\gamma$, the differential along the gauge orbits, acts only on the fields $\Phi^{A}$. Since the initial theory is free, the structure functions $f_{0}{ }^{\alpha}{ }_{\beta \gamma}$ vanish, therefore $\gamma$ only acts on the fields $\varphi^{i}$ of the quadratic action $S_{0}$. Together with $\delta^{2}=0=\gamma^{2}$, the anticommutation relation $\{\delta, \gamma\}=0$ is true. One extends the action of $s$ on derivatives of the fields and antifields by $\{s, d\}=0$, where $d$ is the total exterior derivative

$$
d:=d x^{\mu} \partial_{\mu}=d x^{\mu}\left(\frac{\partial}{\partial x^{\mu}}+\Phi_{\mu}^{A} \frac{\partial^{L}}{\partial \Phi^{A}}+\Phi_{A \mu}^{\star} \frac{\partial^{L}}{\partial \Phi_{A}^{\star}}+\ldots\right),
$$

where $\Phi_{\mu}^{A}=\frac{\partial \Phi^{A}}{\partial x^{\mu}}$, idem for $\Phi_{A \mu}^{\star}$. The Koszul-Tate differential decreases the antifield number antifd by one unit, while the differential $\gamma$ increases the pure ghost number puregh by one unit, so that one has $g h=$ puregh - antifd. The BV functional $W_{0}$ is required to have a definite ghost number and to start with the initial action, $W_{0}=S_{0}+$ antifielddependent terms, therefore $g h\left(W_{0}\right)=0$ since one assigns zero ghost number to the fields $\varphi^{i}$.

In the BRST formalism, the ghosts $C^{\alpha}$ have the opposite Grassmann parity compared to the original gauge parameters $\epsilon^{\alpha}$. In the antifield extension of the BRST formalism, the Grassmann parities of the antifields are given by $\left|\Phi_{A}^{\star}\right|=\left|\Phi^{A}\right|+1$, where $|Z|=0 \bmod 2$ for bosonic fields $Z$, and $|Z|=1 \bmod 2$ for fermionic fields $Z$. Moreover, the ghost number of the antifields is $g h\left(\Phi_{A}^{\star}\right)=-g h\left(\Phi^{A}\right)-1$. One demands that $W_{0}$ be of definite Grassmann parity, therefore $\left|W_{0}\right|=0$.

Infinitesimal deformations. The antifield formalism is also particularly efficient in order to perturbatively deform the initial action [7], $S\left[\varphi^{i}\right]=S_{0}+S_{1}+S_{2}+\ldots$, where $S_{1}$ and $S_{2}$ are respectively linear and quadratic in the collection of infinitesimal parameters, while at the same time deforming the initial gauge variations $\Delta=\Delta_{0}+\Delta_{1}+\Delta_{2}+\ldots$, such that one has $\Delta S=0$. We introduce a set of infinitesimal deformation parameters collectively denoted by $g$ and relative coupling constants $\tilde{\kappa}$ (index omitted for both $g$ and $\tilde{\kappa}$ ) so that the expansion of $S$ is in the $g \tilde{\kappa}$ 's. The requirement of gauge invariance $\Delta S=0$ amounts to asking that $W\left[\Phi^{A}, \Phi_{A}^{\star}\right]=W_{0}\left[\Phi^{A}, \Phi_{A}^{\star}\right]+g W_{1}\left[\Phi^{A}, \Phi_{A}^{\star}\right]+g^{2} W_{2}\left[\Phi^{A}, \Phi_{A}^{\star}\right]+\mathcal{O}\left(g^{3}\right)$ satisfies the master equation (2.2). To first and second orders in the deformation parameters, it means that

$$
\begin{aligned}
\left(W_{0}, W_{1}\right) & =0, \\
\left(W_{0}, W_{2}\right)+\frac{1}{2}\left(W_{1}, W_{1}\right) & =0 .
\end{aligned}
$$

Upon recognising that an infinitesimal deformation $W_{1}$ is trivial if it is of the form $W_{1}=$ $\left(W_{0}, B\right)=s B$ for a local functional $B$ of ghost number -1 , the classification of infinitesimal deformations is a cohomological problem, see [7] and [11] for more details.

We assume that the fields and their derivatives vanish at infinity, so that we do not take boundary terms into account inside the action or its variation. Decomposing the first order infinitesimal deformation $W_{1}$ with respect to the antifield number, general results derived in $[23,24]$ based on the assumption of locality of the deformation allow us to stop at antifield number 2 for the type of theories at hand:

$$
W_{1}=\int d^{4} x\left(a_{0}+a_{1}+a_{2}\right) .
$$


Equation (2.6) then yields a $(\delta, \gamma)$ descent of equations in antifield numbers for the densities $a_{\text {antifd }}$ (from $a_{2}$ to $a_{1}$ and $a_{0}$ ):

$$
\begin{aligned}
\delta a_{1}+\gamma a_{0} & =\partial_{\mu} j_{0}^{\mu}, \\
\delta a_{2}+\gamma a_{1} & =\partial_{\mu} j_{1}^{\mu}, \\
\gamma a_{2} & =0 .
\end{aligned}
$$

That one can redefine away a total derivative from the right-hand side of the last equation also follows from standard arguments [23, 24].

At second order in deformation, for $W_{2}=\int d^{4} x\left(b_{0}+b_{1}+b_{2}\right)$, the equation (2.7) splits into

$$
\begin{aligned}
\delta b_{1}+\gamma b_{0} & =-\left(a_{1}, a_{0}\right)+\partial_{\mu} t_{0}^{\mu}, \\
\delta b_{2}+\gamma b_{1} & =-\frac{1}{2}\left(a_{1}, a_{1}\right)-\left(a_{2}, a_{1}\right)+\partial_{\mu} t_{1}^{\mu}, \\
\gamma b_{2} & =-\frac{1}{2}\left(a_{2}, a_{2}\right)+\partial_{\mu} t_{2}^{\mu} .
\end{aligned}
$$

The form of the right hand sides of these second order equations is restricted by properties of the solutions of the first order ones.

\subsection{First starting point: free BV functionals in flat background}

The BV spectrum of the theories generated by $\mathcal{N}_{4}$ massless Rarita-Schwinger fields and $n_{v}$ vector gauge fields is given by:

- 1 graviton whose gauge potential is $h_{\mu \nu}$, the metric perturbation around the Minkowski background, such that $g_{\mu \nu}=\eta_{\mu \nu}+\kappa h_{\mu \nu}$ is the complete metric. (In the $A d S_{4}$ background, $h_{\mu \nu}$ will refer to the decomposition $g_{\mu \nu}=\bar{g}_{\mu \nu}+\kappa h_{\mu \nu}$ of the dynamical metric around the $A d S_{4}$ background $\bar{g}_{\mu \nu}$.);

- $\mathcal{N}_{4}$ gravitini $\left(\psi_{\mu}^{\Delta}\right)_{A}, \Delta=1, \ldots, \mathcal{N}_{4}$ in the Majorana representation, where we refer to appendix A for the conventions used for 4-component Majorana spinors with index $A$;

- $n_{v}$ Maxwell gauge fields $A_{\mu}^{a}, a=1, \ldots, n_{v}$;

- Zero or one spin- $\frac{1}{2}$ field $(\chi)_{A}$ in the Majorana representation;

- The ghosts $\xi_{\mu}$ associated with linearised diffeomorphisms;

- $\mathcal{N}_{4}$ ghosts $\zeta^{\Delta}$ associated with the spin- $\frac{3}{2}$ gauge symmetry of each Rarita-Schwinger gauge fields;

- $n_{v} \operatorname{ghost}(\mathrm{s}) \mathcal{C}^{a}$ associated with the abelian factors $(\mathrm{U}(1))^{\times n_{v}}$ of the gauge group;

- An antifield $\Phi_{I}^{\star}$ associated with each field or ghost $\Phi^{I}$.

Each of these fields carries a pure ghost number puregh, an antifield number antifd and a ghost number $g h=$ puregh - antifd that are listed in table 1 and in table 2, in which 
we also indicated the action of the differentials $\gamma, \delta$ and $s=\gamma+\delta$ around the flat and $A d S_{4}$ backgrounds.

In Minkowski background, the BV functional of the free theory associated with the sum of the free actions for a massless spin-2, several spin- $\frac{3}{2}$ and spin- 1 fields and one spin- $\frac{1}{2}$ field is given by

$$
\begin{aligned}
W_{0}= & S^{D}\left[\chi_{A}\right]+S^{M}\left[A_{\mu}^{a}\right]+S^{R S}\left[\psi_{\mu A}^{\Delta}\right]+S^{F P}\left[h_{\mu \nu}\right] \\
& +\int d^{4} x A_{a}^{\star \mu} \partial_{\mu} \mathcal{C}^{a}+\int d^{4} x \psi_{\Delta}^{\star \mu A} \partial_{\mu} \zeta_{A}^{\Delta}+\int d^{4} x 2 h^{\star \mu \nu} \partial_{(\mu} \xi_{\nu)},
\end{aligned}
$$

where

$$
\begin{aligned}
S^{D}\left[\chi_{A}\right] & =-\frac{1}{2} \int d^{4} x \bar{\chi}^{A}\left(\gamma^{\mu}\right)_{A}{ }^{B} \partial_{\mu} \chi_{B} \\
S^{M}\left[A_{\mu}^{a}\right] & =-\frac{1}{4} \int d^{4} x F_{\mu \nu}^{a} F^{b \mu \nu} \delta_{a b}, \quad F_{\mu \nu}^{a}:=\partial_{\mu} A_{\nu}^{a}-\partial_{\nu} A_{\mu}^{a} \\
S^{R S}\left[\psi_{\mu A}^{\Delta}\right] & =-\frac{1}{2} \int d^{4} x \bar{\psi}_{\mu}^{\Delta A}\left(\gamma^{\mu \nu \rho}\right)_{A}{ }^{B} \partial_{\nu} \psi_{\rho B}^{\Omega} \delta_{\Delta \Omega} \\
S^{F P}\left[h_{\mu \nu}\right] & =\int d^{4} x\left(-\frac{1}{2} \partial_{\mu} h_{\nu \rho} \partial^{\mu} h^{\nu \rho}+\partial_{\mu} h_{\nu}^{\mu} \partial_{\rho} h^{\rho \nu}-\partial_{\nu} h_{\mu}{ }^{\mu} \partial_{\rho} h^{\rho \nu}+\frac{1}{2} \partial_{\mu} h_{\nu}{ }^{\nu} \partial^{\mu} h_{\rho}{ }^{\rho}\right) .
\end{aligned}
$$

The field equations for the Fierz-Pauli gauge field $h_{\mu \nu}$ are denoted by $-2 \stackrel{(1)}{G}^{\mu \nu} \approx 0$, where weak equality means "on the surface of the solutions to the free field equations" and where the linearised Einstein tensor is given by

$$
\stackrel{(1)}{G}^{\mu \nu}:=-\frac{1}{2}\left(\square h^{\mu \nu}-\partial^{\mu} \partial_{\rho} h^{\rho \nu}-\partial^{\nu} \partial_{\rho} h^{\rho \mu}+\partial^{\mu} \partial^{\nu} h+\eta^{\mu \nu} \partial_{\rho} \partial_{\sigma} h^{\rho \sigma}-\eta^{\mu \nu} \square h\right) .
$$

The action of the BRST differential acting on each field and antifield is summarised in table 1. A central cohomological group for the deformation procedure of the initial theory is $H(\gamma)$, the cohomology of $\gamma$ in the space of local functions. Defining the field strength

$$
K_{\alpha \beta \mid \mu \nu}:=-\partial_{\alpha} \partial_{[\mu} h_{\nu] \beta}+\partial_{\beta} \partial_{[\mu} h_{\nu] \alpha},
$$

$H(\gamma)$ is given by

$$
H(\gamma) \cong\left\{f\left(\left[\chi_{A}\right],\left[F_{\mu \nu}^{a}\right],\left[\partial_{[\mu} \psi_{\nu] A}^{\Delta}\right],\left[K_{\alpha \beta \mid \mu \nu}\right], \mathcal{C}^{a}, \zeta_{A}^{\Delta}, \xi_{\mu}, \partial_{[\mu} \xi_{\nu]},\left[\Phi_{I}^{\star}\right]\right)\right\}
$$

where the notation $[\Phi]$ means the field $\Phi$ and all its derivatives up to some finite, but arbitrary, order. The almost complete elimination of the derivatives of the ghosts and the appearance of gauge invariant expressions follow from the existence of corresponding contractible pairs for contracting homotopies. For vector fields, the cohomology $H(\gamma)$ was computed long ago. We refer the reader to [24] for details and references. In the massless spin-2 case, the cohomology of $\gamma$ was computed in [11], while in the presence of a RaritaSchwinger gauge field, it was given in [21]. 


\begin{tabular}{|c|c|c|c|c|c|c|c|}
\hline & $||$. & $g h$ & puregh & antifd & $\gamma$ & $\delta$ & $s=\gamma+\delta$ \\
\hline \hline$\chi_{A}$ & 1 & 0 & 0 & 0 & 0 & 0 & 0 \\
\hline$A_{\mu}^{a}$ & 0 & 0 & 0 & 0 & $\partial_{\mu} \mathcal{C}^{a}$ & 0 & $\partial_{\mu} \mathcal{C}^{a}$ \\
\hline$\psi_{\mu A}^{\Delta}$ & 1 & 0 & 0 & 0 & $-\partial_{\mu} \zeta_{A}^{\Delta}$ & 0 & $-\partial_{\mu} \zeta_{A}^{\Delta}$ \\
\hline$h_{\mu \nu}$ & 0 & 0 & 0 & 0 & $2 \partial_{\mu} \xi_{\nu)}$ & 0 & $2 \partial_{(\mu} \xi_{\nu}$ \\
\hline \hline $\mathcal{C}^{a}$ & 1 & 1 & 1 & 0 & 0 & 0 & 0 \\
\hline$\zeta_{A}^{\Delta}$ & 0 & 1 & 1 & 0 & 0 & 0 & 0 \\
\hline$\xi_{\mu}$ & 1 & 1 & 1 & 0 & 0 & 0 & 0 \\
\hline \hline$\chi^{\star A}$ & 0 & -1 & 0 & 1 & 0 & $\partial_{\mu} \bar{\chi}^{B}\left(\gamma^{\mu}\right)_{B}{ }^{A}$ & $\partial_{\mu} \bar{\chi}^{B}\left(\gamma^{\mu}\right)_{B}{ }^{A}$ \\
\hline$A_{a}^{\star \mu}$ & 1 & -1 & 0 & 1 & 0 & $\partial_{\nu} F_{a}^{\nu \mu}$ & $\partial_{\nu} F_{a}^{\nu \mu}$ \\
\hline$\psi_{\Delta}^{\star \mu A}$ & 0 & -1 & 0 & 1 & 0 & $-\partial_{\nu} \bar{\psi}_{\Delta \rho}^{B}\left(\gamma^{\mu \nu \rho}\right)_{B}{ }^{A}$ & $-\partial_{\nu} \bar{\psi}_{\Delta \rho}^{B}\left(\gamma^{\mu \nu \rho}\right)_{B}{ }^{A}$ \\
\hline$h^{\star \mu \nu}$ & 1 & -1 & 0 & 1 & 0 & $-2 G^{\mu \nu}$ & $-2 G^{\mu \nu}$ \\
\hline \hline $\mathcal{C}_{a}^{\star}$ & 0 & -2 & 0 & 2 & 0 & $-\partial_{\mu} A_{a}^{\star \mu}$ & $-\partial_{\mu} A_{a}^{\star \mu}$ \\
\hline$\zeta_{\Delta}^{\star A}$ & 1 & -2 & 0 & 2 & 0 & $-\partial_{\mu} \psi_{\Delta}^{\star \mu A}$ & $-\partial_{\mu} \psi_{\Delta}^{\star \mu A}$ \\
\hline$\xi^{\star \mu}$ & 0 & -2 & 0 & 2 & 0 & $-2 \partial_{\nu} h^{\star \mu \nu}$ & $-2 \partial_{\nu} h^{\star \mu \nu}$ \\
\hline
\end{tabular}

Table 1. Grassmann parity, ghost number, pureghost number, antifield number and actions of the differentials $\gamma, \delta$ and $s$ on the various fields of the spectrum, for the theory around flat background.

\subsection{Second starting point: anti de Sitter background}

The components of the background $A d S_{4}$ vierbeins and spin connection are denoted by $\bar{e}_{\mu}{ }^{a}$ and $\bar{\omega}_{\mu}^{a b}$. The components of the background $A d S_{4}$ metric therefore read $\bar{g}_{\mu \nu}=\bar{e}_{\mu}{ }^{a} \bar{e}_{\nu}{ }^{b} \eta_{a b}$. We denote the Lorentz-covariant derivative on $A d S_{4}$ by $\nabla_{\mu}$ and the corresponding one-form differential operator $\nabla=d x^{\mu} \nabla_{\mu}$. Introducing $\sigma_{a b}=\frac{1}{2} \gamma_{a b}$, then $i \sigma_{a b}$ gives a representation of the Lorentz algebra on the spinor representation and $\nabla \psi=d \psi+\Omega \psi$ where $\Omega:=\frac{1}{4} \bar{\omega}^{a b} \gamma_{a b}$. On the conjugate spinor $\bar{\psi}$, the Lorentz-covariant derivative acts like $\nabla \bar{\psi}=d \bar{\psi}-\bar{\psi} \Omega$. Our conventions are such that the commutator of Lorentz-covariant derivatives on $A d S_{4}$, when acting on a vector or on a spinor, is given by

$$
\left[\nabla_{\mu}, \nabla_{\nu}\right] \xi_{\sigma}=-2 \lambda^{2} \bar{g}_{\sigma[\mu} \xi_{\nu]}, \quad\left[\nabla_{\mu}, \nabla_{\nu}\right] \zeta_{A}^{\Delta}=-\lambda^{2}\left(\sigma_{\mu \nu}\right)_{A}{ }^{B} \zeta_{B}^{\Delta}
$$

where $\sigma_{\mu \nu}:=\bar{e}_{\mu}{ }^{a} \bar{e}_{\nu}{ }^{b} \sigma_{a b}, \lambda$ is the $A d S_{4}$ inverse radius that, in four spacetime dimension, is related to the cosmological constant $\Lambda$ by $\Lambda=-3 \lambda^{2}$.

In $A d S_{4}$ background, the BV functional of the free theory associated with the sum of the free actions for a massless spin- 2 , several spin- $\frac{3}{2}$ and spin- 1 fields and one spin- $\frac{1}{2}$ field is given by

$$
\begin{aligned}
W_{0}^{\Lambda}= & S_{\Lambda}^{D}\left[\chi_{A}\right]+S_{\Lambda}^{M}\left[A_{\mu}^{a}\right]+S^{R S}\left[\psi_{\mu A}^{\Delta}\right]+S_{\Lambda}^{F P}\left[h_{\mu \nu}\right]+\int d^{4} x \sqrt{-\bar{g}} A_{a}^{\star \mu} \nabla_{\mu} \mathcal{C}^{a} \\
& +\int d^{4} x \sqrt{-\bar{g}} \psi_{\Delta}^{\star \mu A}\left(\nabla_{\mu} \zeta_{A}^{\Delta}+\frac{\lambda}{2}\left(\gamma_{\mu}\right)_{A}{ }^{B} \zeta_{B}^{\Delta}\right)+\int d^{4} x \sqrt{-\bar{g}} 2 h^{\star \mu \nu} \nabla_{(\mu} \xi_{\nu)},
\end{aligned}
$$


where

$$
\begin{aligned}
S_{\Lambda}^{D}\left[\chi_{A}\right] & =-\frac{1}{2} \int d^{4} x \sqrt{-\bar{g}} \bar{\chi}^{A}\left(\gamma^{\mu}\right)_{A}{ }^{B} \nabla_{\mu} \chi_{B}, \\
S_{\Lambda}^{M}\left[A_{\mu}^{a}\right] & =-\frac{1}{4} \int d^{4} x \sqrt{-\bar{g}} F_{\mu \nu}^{a} F^{b \mu \nu} \delta_{a b}, \\
S_{\Lambda}^{R S}\left[\psi_{\mu A}^{\Delta}\right] & =\int d^{4} x \sqrt{-\bar{g}}\left(-\frac{1}{2} \bar{\psi}_{\mu}^{\Delta A}\left(\gamma^{\mu \nu \rho}\right)_{A}{ }^{B} \nabla_{\nu} \psi_{\rho B}^{\Omega}+\frac{\lambda}{2} \bar{\psi}_{\mu}^{\Delta A}\left(\gamma^{\mu \nu}\right)_{A}{ }^{B} \psi_{\nu B}^{\Omega}\right) \delta_{\Delta \Omega}, \\
S_{\Lambda}^{F P}\left[h_{\mu \nu}\right] & =\int d^{4} x \sqrt{-\bar{g}}\left(-\frac{1}{2} \nabla_{\mu} h_{\nu \rho} \nabla^{\mu} h^{\nu \rho}+\nabla_{\mu} h_{\nu}^{\mu} \nabla_{\rho} h^{\rho \nu}-\nabla_{\nu} h_{\mu}{ }^{\mu} \nabla_{\rho} h^{\rho \nu}\right. \\
& \left.+\frac{1}{2} \nabla_{\mu} h_{\nu}{ }^{\nu} \nabla^{\mu} h_{\rho}{ }^{\rho}+\frac{\lambda^{2}}{2}\left(2 h_{\mu \nu} h^{\mu \nu}+h_{\mu}{ }^{\mu} h_{\nu}{ }^{\nu}\right)\right) .
\end{aligned}
$$

The gauge-invariant field strengths for the spin-1, spin- $\frac{3}{2}$ and spin-2 fields are

$$
\begin{aligned}
F_{\mu \nu}^{a}:= & \nabla_{\mu} A_{\nu}^{a}-\nabla_{\nu} A_{\mu}^{a}, \quad \Psi_{\mu \nu A}^{\Delta}:=\nabla_{[\mu} \psi_{\nu] A}^{\Delta}+\frac{\lambda}{2}\left(\gamma_{[\mu}\right)_{A}{ }^{B} \psi_{\nu] B}^{\Delta}, \\
\tilde{K}^{\alpha \beta \mid \mu \nu}:= & -\frac{1}{2}\left(\nabla^{\alpha} \nabla^{[\mu} h^{\nu] \beta}-\nabla^{\beta} \nabla^{[\mu} h^{\nu] \alpha}+\nabla^{\mu} \nabla^{[\alpha} h^{\beta] \nu}-\nabla^{\nu} \nabla^{[\alpha} h^{\beta] \mu}\right) \\
& +\lambda^{2}\left(\bar{g}^{\alpha[\mu} h^{\nu] \beta}-\bar{g}^{\beta[\mu} h^{\nu] \alpha}\right) .
\end{aligned}
$$

When considering the $A d S_{4}$ background, we will denote by $-2 \tilde{G}^{\mu \nu}$ the left-hand side of the Euler-Lagrange equations for the Fierz-Pauli gauge field $h_{\mu \nu}$. The tensor $\tilde{G}^{\mu \nu}$ is the linearisation around $A d S_{4}$ of $G_{\mu \nu}+\Lambda g_{\mu \nu}$. It is given by

$$
\begin{gathered}
\tilde{G}^{\mu \nu}=-\frac{1}{2}\left(\bar{\square} h^{\mu \nu}-\nabla^{\mu} \nabla_{\sigma} h^{\sigma \nu}-\nabla^{\nu} \nabla_{\sigma} h^{\sigma \mu}+\nabla^{\mu} \nabla^{\nu} h+\bar{g}^{\mu \nu} \nabla_{\rho} \nabla_{\sigma} h^{\rho \sigma}\right. \\
\left.-\bar{g}^{\mu \nu} \bar{\square} h+2 \lambda^{2} h^{\mu \nu}+\lambda^{2} \bar{g}^{\mu \nu} h\right),
\end{gathered}
$$

where $\bar{\square}:=\bar{g}^{\mu \nu} \nabla_{\mu} \nabla_{\nu}$ is the Laplace-Beltrami operator in $A d S_{4}$ and $h:=h_{\mu \nu} \bar{g}^{\mu \nu}$. The action of the BRST differential acting on each field and antifield is summarised in table 2. Note that, in this table as well as in the action, we have not written any mass term for the real spin- $1 / 2$ field. This is not a loss of generality but a result that is explained in footnote 6 .

Finally, along similar lines to those followed in flat space, we find that the cohomology of $\gamma$ is

$$
H(\gamma) \cong\left\{f\left(\left[\chi_{A}\right],\left[F_{\mu \nu}^{a}\right],\left[\Psi_{\mu \nu A}^{\Delta}\right],\left[\tilde{K}^{\alpha \beta \mid \mu \nu}\right], \mathcal{C}^{a}, \zeta_{A}^{\Delta}, \xi_{\mu}, \nabla_{[\mu} \xi_{\nu]},\left[\Phi_{I}^{\star}\right]\right)\right\}
$$

\section{Uniqueness of $\mathcal{N}_{4}=2$ and $\mathcal{N}_{4}=3$ pure supergravities}

In this section we first give, in subsection 3.1, the classification of the most general, consistent cubic vertices for the initial theory describing the free propagation, around the flat background, of one graviton, two massless Rarita-Schwinger fields and one Maxwell gauge field. The $\mathcal{N}_{4}=2$ pure supergravity theory (without cosmological constant) [19] is recovered as the unique theory that deforms the gauge algebra away from the abelian algebra of 


\begin{tabular}{|c|c|c|c|c|c|c|}
\hline & $|\cdot|$ & $g h$ & puregh & antifd & $\frac{1}{\sqrt{-\bar{g}}} \gamma$ & $\frac{1}{\sqrt{-\bar{g}}} \delta$ \\
\hline$\chi_{A}$ & 1 & 0 & 0 & 0 & 0 & 0 \\
\hline$A_{\mu}^{a}$ & 0 & 0 & 0 & 0 & $\nabla_{\mu} \mathcal{C}^{a}$ & 0 \\
\hline$\psi_{\mu A}^{\Delta}$ & 1 & 0 & 0 & 0 & $-\nabla_{\mu} \zeta_{A}^{\Delta}-\frac{\lambda}{2}\left(\gamma_{\mu}\right)_{A}^{B} \zeta_{B}^{\Delta}$ & 0 \\
\hline$h_{\mu \nu}$ & 0 & 0 & 0 & 0 & $2 \nabla_{(\mu} \xi_{\nu)}$ & 0 \\
\hline $\mathcal{C}^{a}$ & 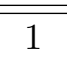 & 1 & 1 & 0 & 0 & 0 \\
\hline$\zeta_{A}^{\Delta}$ & 0 & 1 & 1 & 0 & 0 & 0 \\
\hline$\xi_{\mu}$ & 1 & 1 & 1 & 0 & 0 & 0 \\
\hline$\chi^{\star A}$ & 0 & -1 & 0 & 1 & 0 & $\nabla_{\mu} \bar{\chi}^{B}\left(\gamma^{\mu}\right)_{B}{ }^{A}$ \\
\hline$A_{a}^{\star \mu}$ & 1 & -1 & 0 & 1 & 0 & $\nabla_{\nu} F_{a}^{\nu \mu}$ \\
\hline$\psi_{\Delta}^{\star \mu A}$ & 0 & -1 & 0 & 1 & 0 & $-\nabla_{\nu} \bar{\psi}_{\Delta \rho}^{B}\left(\gamma^{\mu \nu \rho}\right)_{B}{ }^{A}-\lambda \bar{\psi}_{\Delta \nu}^{B}\left(\gamma^{\mu \nu}\right)_{B}{ }^{A}$ \\
\hline$h^{\star \mu \nu}$ & 1 & -1 & 0 & 1 & 0 & $-2 \tilde{G}^{\mu \nu}$ \\
\hline $\mathcal{C}_{a}^{\star}$ & 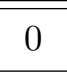 & -2 & 0 & 2 & 0 & $-\nabla_{\mu} A_{a}^{\star \mu}$ \\
\hline$\zeta_{\Delta}^{\star A}$ & 1 & -2 & 0 & 2 & 0 & $-\nabla_{\mu} \psi_{\Delta}^{\star \mu A}+\frac{\lambda}{2} \psi_{\Delta}^{\star \mu B}\left(\gamma_{\mu}\right)_{B}{ }^{A}$ \\
\hline$\xi^{\star \mu}$ & 0 & -2 & 0 & 2 & 0 & $-2 \nabla_{\nu} h^{\star \mu \nu}$ \\
\hline
\end{tabular}

Table 2. Grassmann parity, ghost number, pureghost number, antifield number and actions of the differentials $\gamma$ and $\delta$ on the various fields of the spectrum, for the theory around $A d S_{4}$ background.

the initial, free theory. In this model, the gravitini are not charged under the U(1) gauge field of electromagnetism. The well-known obstruction $[1,2]$ to the minimal coupling of the gravitini around Minkowski spacetime is rederived in cohomological terms in subsection 3.2. Most of the technical details of the computations concerning subsection 3.1 are not given. They can be found in [27], and some of them will be given later in the next subsection 3.3, where we first deform the theory by changing background from flat spacetime to $A d S_{4}$, and then further deform the theory until the actions of pure $\mathcal{N}_{4}=2$ and $\mathcal{N}_{4}=3$ supergravities with cosmological constant terms [17] are obtained.

\subsection{Uniqueness of $\mathcal{N}=2$ pure sugra}

In this subsection, the single Latin index labelling the Maxwell gauge field will be omitted. We introduce no Majorana spin- $\frac{1}{2}$ field $\chi$. The upper-case Greek indices labelling the various gravitini run from 1 to 2 and are raised and lowered with the Euclidean metric in the internal space of the gravitini.

First order deformation. In order to solve the last equation (2.11) of the descent for the algebra-deforming cubic candidates denoted by $a_{2}$, we have to classify all the $a_{2} \in H(\gamma)$ that are cubic, i.e., of the type " $C^{\star} C C$ " since, in (2.1), the term $\frac{1}{2} C_{\alpha}^{\star} f_{0}{ }^{\alpha}{ }_{\beta \gamma} C^{\beta} C^{\gamma}$ encodes the information about the gauge algebra. By using the result on the cohomology of $\gamma$ recalled in (2.22), all the possible $a_{2}$ 's that are parity and Poincaré invariant are listed 
as follows:

$$
\begin{array}{llrl}
a_{2}^{(1)}=\xi^{\star \mu} \xi^{\nu} \partial_{[\mu} \xi_{\nu]}, & a_{2}^{(2)}=\frac{1}{4} k_{\Delta \Omega}^{(2)} \xi^{\star \mu} \bar{\zeta}^{(\Delta} \gamma_{\mu} \zeta^{\Omega)}, & k_{\Delta \Omega}^{(2)}=k_{(\Delta \Omega)}^{(2)}, \\
a_{2}^{(3)}=\xi^{\star \mu} \xi_{\mu} \mathcal{C}, & a_{2}^{(4)}=k_{\Delta \Omega}^{(4)} \mathcal{C}^{\star} \bar{\zeta}^{[\Delta} \zeta^{\Omega]}, & k_{\Delta \Omega}^{(4)}=k_{[\Delta \Omega]}^{(4)}, & a_{2}^{(5)}=k_{\Delta \Omega}^{(5)} \zeta^{\star \Delta} \zeta^{\Omega} \mathcal{C}, \\
a_{2}^{(6)}=k_{\Delta \Omega}^{(6)} \zeta^{\star \Delta} \gamma^{\mu} \zeta^{\Omega} \xi_{\mu}, & a_{2}^{(7)}=k_{\Delta \Omega}^{(7)} \zeta^{\star \Delta} \gamma^{\mu \nu} \zeta^{\Omega} \partial_{[\mu} \xi_{\nu]} . &
\end{array}
$$

Thus the most general gauge-algebra deformation at first order, is a priori a linear combination of these seven candidates with the same number of associated infinitesimal deformation parameters.

The next step amounts to solving equation (2.10) for the $a_{1}$ 's. A direct computation gives

$$
\begin{array}{ll}
a_{1}^{(1)}=h^{\star \mu \nu}\left[\partial_{\mu} \xi^{\sigma} h_{\nu \sigma}-\xi^{\sigma}\left(\partial_{\nu} h_{\nu \sigma}-\partial_{\sigma} h_{\mu \nu}\right)\right], & a_{1}^{(2)}=-k_{\Delta \Omega}^{(2)} h^{\star \mu \nu} \bar{\psi}_{\mu}^{\Delta} \gamma_{\nu} \zeta^{\Omega}, \\
a_{1}^{(3)}=h^{\star \mu \nu}\left(2 A_{\mu} \xi_{\nu}-h_{\mu \nu} \mathcal{C}\right), & a_{1}^{(4)}=-2 k_{\Delta \Omega}^{(4)} A^{\star \mu} \bar{\psi}_{\mu}^{\Delta} \zeta^{\Omega} \\
a_{1}^{(5)}=k_{\Delta \Omega}^{(5)} \psi^{\star \mu \Delta}\left(\psi_{\mu}^{\Omega} \mathcal{C}-\zeta^{\Omega} A_{\mu}\right), & \\
a_{1}^{(7)}=\psi^{\star \Delta \rho} k_{\Delta \Omega}^{(7)} \gamma^{\mu \nu}\left(\psi_{\rho}^{\Omega} \partial_{[\mu} \xi_{\nu]}-\zeta^{\Omega} \partial_{[\mu} h_{\nu] \rho}\right) . &
\end{array}
$$

Equation (2.10) has no nontrivial solution for $a_{1}^{(6)}$ as a function of $a_{2}=a_{2}^{(6)}$, therefore we have to set to zero the coefficient in front of it, in the linear combination of the seven $a_{2}$ 's. Indeed, we find

$$
\delta a_{2}^{(6)}=\partial_{\mu} j_{1}^{\mu}-\gamma\left(k_{\Delta \Omega}^{(6)} \psi^{\star \Delta \rho} \gamma_{\mu} \psi_{\rho}^{\Omega} \xi^{\mu}-\frac{1}{2} k_{\Delta \Omega}^{(6)} \psi^{\star \Delta \rho} \gamma^{\mu} \zeta^{\Omega} h_{\mu \rho}\right)+k_{\Delta \Omega}^{(6)} \psi^{\star \Delta \rho} \gamma^{\mu} \zeta^{\Omega} \partial_{[\rho} \xi_{\mu]}
$$

and observe that the last term in the above equation is in $H(\gamma)$ and therefore represents an obstruction to finding a solution for $a_{1}^{(6)}$. Note that the solution for the six elements $a_{1}$ found above in (3.4)-(3.7) can be supplemented by the general solution of the homogeneous equation

$$
\gamma a_{1}=0
$$

where a total derivative term in the right-hand side has been absorbed by a trivial redefinition, as done e.g. in [11, 21]. In the following, we will use the notation $\hat{a}_{1}$ to indicate antifield number 1 cocycles at ghost number zero that obey (3.9) and can be lifted to an $a_{0}$, i.e., such that $\delta \hat{a}_{1}+\gamma a_{0}=\partial_{\mu} \beta^{\mu}$ is true for some $a_{0}$ and $\beta^{\mu}$. In other words such an $\hat{a}_{1}$ provides a solution of the descent equations of subsection 2.1 that does not contain an $a_{2}$ term, i.e, that does not deform the gauge algebra. On the other hand, we will use the notation $\tilde{a}_{1}$ for antifield number 1 cocycles at ghost number zero that obey (3.9) and that are necessary to add to a candidate $a_{1}$ that comes from a corresponding $a_{2}$, when an obstruction arises in lifting $a_{1}$ alone.

We now turn to the resolution of equation (2.9) for the candidate cubic vertices associated with the linear combination of the above six $a_{1}$ 's. The resolution of equation (2.9) with $a_{1}=a_{1}^{(1)}$ as source can be done separately and gives the cubic part of the EinsteinHilbert action, as was done in [11]. The resolution of (2.9) with $a_{1}^{(2)}$ and $a_{1}^{(7)}$ as sources 
has to be done concurrently, as these two terms talk to each other in the solution for the vertex and do not mix with the others. It turns out, as was observed in [21], that in order to find a vertex $a_{0}$ from the two sources $a_{1}^{(2)}$ and $a_{1}^{(7)}$, one must add to them the following solution of the homogeneous equation (3.9):

$$
\tilde{a}_{1}^{(2-7)}=\psi^{\star \Delta \mu} \tilde{k}_{\Delta \Omega} \partial_{[\mu} \psi_{\nu]}^{\Omega} \xi^{\nu} .
$$

Then, by use of $\gamma\left(\partial_{[\nu} h_{\rho] \mu}\right)=\partial_{\mu} \partial_{[\nu} \xi_{\rho]}$ and the relation (A.3), equation (2.9) admits a nontrivial solution if and only if

$$
k_{\Delta \Omega}^{(2)}=k_{\Delta \Omega}^{(7)}=-\frac{1}{8} \tilde{k}_{\Delta \Omega}=: k_{\Delta \Omega},
$$

implying that $k_{\Delta \Omega}^{(7)}$ and $\tilde{k}_{\Delta \Omega}$ both inherit the symmetry of $k_{\Delta \Omega}^{(2)}: k_{\Delta \Omega}=k_{(\Delta \Omega)}$. The solution for the corresponding cubic vertices explicitly reads

$$
\begin{aligned}
a_{0}^{(2-7)}= & \bar{\psi}_{\mu}^{\Delta} \gamma^{\mu \nu \rho}\left(\sigma^{\alpha \beta} \partial_{[\alpha} h_{\beta] \nu}\right) \psi_{\rho}^{\Omega} k_{\Delta \Omega}-h \bar{\psi}_{\mu}^{\Delta} \gamma^{\mu \nu \rho} \partial_{\nu} \psi_{\rho}^{\Omega} k_{\Delta \Omega} \\
& +h_{\alpha \beta} \bar{\psi}^{\alpha \Delta} \gamma^{\beta \nu \rho} \partial_{\nu} \psi_{\rho}^{\Omega} k_{\Delta \Omega}+h_{\alpha \beta} \bar{\psi}_{\mu}^{\Delta} \gamma^{\mu \alpha \nu} \partial^{\beta} \psi_{\rho}^{\Omega} k_{\Delta \Omega}+h_{\alpha \beta} \bar{\psi}_{\mu}^{\Delta} \gamma^{\mu \nu \alpha} \partial_{\nu} \psi^{\beta \Omega} k_{\Delta \Omega} .
\end{aligned}
$$

Continuing with the solution of (2.9) starting from the other sources given in (3.4)-(3.7), we see that the source $a_{1}^{(3)}$ presents an obstruction to admitting a corresponding cubic vertex $a_{0}^{(3)}$. Indeed, a potential solution $a_{0}^{(3)}$ has to be linear in $A_{\mu}$, quadratic in $h_{\mu \nu}$ and must possess one derivative. All the Poincaré-invariant possibilities are covered by $a_{0}^{(3)}=\sum_{i=1}^{6} x_{i} b^{i}, x_{i} \in \mathbb{R}$, where

$$
\begin{array}{lll}
b^{1}=A^{\mu} \partial_{\mu} h^{\alpha \beta} h_{\alpha \beta}, & b^{2}=A^{\mu} \partial_{\mu} h h, & b^{3}=A^{\mu} \partial_{\alpha} h_{\mu \beta} h^{\alpha \beta}, \\
b^{4}=A^{\mu} \partial^{\alpha} h_{\mu \alpha} h, & b^{5}=A_{\mu} \partial_{\alpha} h h^{\mu \alpha}, & b^{6}=A^{\mu} \partial_{\beta} h^{\beta \alpha} h_{\alpha \mu} .
\end{array}
$$

A direct computation shows that there is no possible choice for the coefficients $x_{i}$ that can solve (2.9). To cure this situation one cannot resort to an $\tilde{a}_{1}^{(3)}$ solution of the homogeneous equation (3.9), because an element of $H(\gamma)$ that has the right structure in terms of fields, ghosts and antifields will necessarily bring too many derivatives and therefore cannot mix up with $\delta a_{1}^{(3)}$ to solve (2.9) with $a_{1}^{(3)}+\tilde{a}_{1}^{(3)}$ as source for $a_{0}^{(3)}$.

We now continue the process and consider $a_{1}^{(4)}$ as source for a potential vertex $a_{0}^{(4)}$. By adding

$$
\tilde{a}_{1}^{(4)}:=\tilde{t}_{\Delta \Omega} \psi_{\sigma}^{\star \Delta} F_{\mu \nu} \gamma^{\mu \nu} \gamma^{\sigma} \zeta^{\Omega}
$$

to $a_{1}^{(4)}$, it becomes possible to solve (2.9) for an $a_{0}^{(4)}$, if and only if

$$
\tilde{t}_{\Delta \Omega}=\frac{1}{2} k_{\Delta \Omega}^{(4)}=: t_{\Delta \Omega}
$$

therefore we have that $t_{\Delta \Omega}=t_{[\Delta \Omega]}$ must be antisymmetric because $k_{\Delta \Omega}^{(4)}$ has this property. In particular, this proves the impossibility to couple, minimally or not, a single Majorana 
spin- $\frac{3}{2}$ gauge field to electromagnetism, as was announced in the abstract and in the introduction. Furthermore, since the upper-case Greek indices run over only two values, we take

$$
t_{\Delta \Omega}=\epsilon_{\Delta \Omega}
$$

the $\mathfrak{s p}_{2}$-invariant antisymmetric symbol, up to a coefficient that we will insert when considering a linear combination of all the $a_{1}$ 's. By using the relations (A.4), (A.5), (A.7) and (A.8) the cubic vertex $a_{0}^{(4)}$ that we obtain reads

$$
a_{0}^{(4)}=\epsilon_{\Delta \Omega} \bar{\psi}_{\mu}^{\Delta}\left(F^{\mu \nu} \mathbb{1}-i \gamma^{5}(* F)^{\mu \nu}\right) \psi_{\nu}^{\Omega}, \quad(* F)^{\mu \nu}:=\frac{1}{2} \epsilon^{\mu \nu \rho \sigma} F_{\rho \sigma},
$$

where $\epsilon^{\mu \nu \rho \sigma}$ is the tensorial quantity such that $\epsilon^{0123}=1$ in Cartesian coordinates. This cubic vertex is part of the interactions of the $\mathcal{N}_{4}=2$ pure supergravity Lagrangian of [19] and is a Pauli dipolar coupling.

Finally, when trying to solve (2.9) for a possible vertex $a_{0}^{(5)}$, with $a_{1}^{(5)}$ as the source, we find an obstruction. This is the well-known problem $[1,2]$ of the minimal coupling of gravitini to electromagnetism around flat space. We defer the detailed cohomological derivation of this no-go result to the next subsection.

Before doing this, we want to mention that the coupling of the vector field to gravity is produced by the following $\hat{a}_{1}^{G 1}$, solution of (3.9):

$$
\hat{a}_{1}^{G 1}=-A^{\star \mu} F_{\mu \nu} \xi^{\nu} .
$$

This cocycle of $\gamma$ gives rise to the following vertex

$$
a_{0}^{G 1}=-\frac{1}{8} h F^{\mu \nu} F_{\mu \nu}-\frac{1}{2} F^{\mu \sigma} F_{\sigma}{ }^{\nu} .
$$

This vertex does not deform the gauge algebra as it arises from the candidate $\hat{a}_{1}^{G 1}$, solution of (3.9) without $\delta a_{2}$ source term.

Summary at first order. We gather the various results we obtained so far at first order in the deformation parameters:

$$
\begin{aligned}
& a_{2}=\kappa a_{2}^{(1)}+\alpha\left(a_{2}^{(2)}+a_{2}^{(7)}\right)+y a_{2}^{(4)}, \\
& a_{1}=\kappa a_{1}^{(1)}+\alpha\left(a_{1}^{(2)}+a_{1}^{(7)}+\tilde{a}_{1}^{(2-7)}\right)+y\left(a_{1}^{(4)}+\tilde{a}_{1}^{(4)}\right)+\beta \hat{a}_{1}^{G 1}, \\
& a_{0}=\kappa a_{0}^{(1)}+\alpha a_{0}^{(2-7)}+y a_{0}^{(4)}+\beta a_{0}^{G 1},
\end{aligned}
$$

where $\kappa, \alpha, \beta$ and $y$ are (yet unrelated) infinitesimal deformation parameters that constitute the set of parameters that we collectively denoted by $g$ above. The cocycles $a_{2}^{(1)}, a_{2}^{(2)}$, $a_{2}^{(7)}$ and $a_{2}^{(4)}$ are given in (3.1)-(3.3). They encode the first-order deformations of the gauge algebra and have an antifield number two: antifd $\left(a_{2}\right)=2$. For example, $\kappa a_{2}^{(1)}=$ $\kappa \xi^{\star \mu} \xi^{\nu} \partial_{[\mu} \xi_{\nu]}$ gives the Lie algebra of vector fields equipped with the Lie bracket. Indeed, adding to it the trivial piece $\gamma\left(\frac{\kappa}{2} \xi^{\star \mu} \xi^{\nu} h_{\mu \nu}\right)$ gives an equivalent element $a_{2}:=\kappa \xi^{\star \mu} \xi^{\nu} \partial_{\nu} \xi_{\mu}$ from which we read the result announced and produced in [21]. The cocycles $a_{1}^{(1)}, a_{1}^{(2)}$, 
$a_{1}^{(7)}$ and $a_{1}^{(4)}$ are given in $(3.4)-(3.7)$, while $\tilde{a}_{1}^{(2-7)}, \tilde{a}_{1}^{(4)}$ and $\hat{a}_{1}^{G 1}$ are respectively given in (3.10), (3.14) and (3.18). These cocycles $a_{1}$ 's encode the deformations of the gauge transformations at first order and have antifd $=1$. Finally, the corresponding vertices are given in (3.12), (3.17) and (3.19). They have zero antifield number: antifd( $\left.a_{0}\right)=0$.

Second order deformations. We are going to investigate whether the first order deformations can allow for second order extensions. In order to do this, we first solve (2.14) for a deformation of the gauge algebra, $b_{2}$, to second order in the parameters. By means of $\gamma\left(\partial_{[\nu} h_{\rho] \mu}\right)=\partial_{\mu} \partial_{[\nu} \xi_{\rho]},($ A.6), (A.7), (A.8) and the Fierz identity

$k_{\Delta \Sigma} k_{\Omega \Gamma}\left(\zeta^{\star \Delta} \zeta^{\Omega}\right)\left(\bar{\zeta}^{\Sigma} \zeta^{\Gamma}\right)=-\frac{1}{4} k_{\Delta \Sigma} k_{\Omega \Gamma}\left(\zeta^{\star \Delta} \gamma_{\mu} \zeta^{\Sigma}\right)\left(\bar{\zeta}^{\Omega} \gamma^{\mu} \zeta^{\Gamma}\right)+\frac{1}{8} k_{\Delta \Sigma} k_{\Omega \Gamma}\left(\zeta^{\star \Delta} \gamma_{\mu \nu} \zeta^{\Sigma}\right)\left(\bar{\zeta}^{\Omega} \gamma^{\mu \nu} \zeta^{\Gamma}\right)$,

we find that all obstructions to finding $b_{2}$ are cancelled if and only if

$$
\alpha=\frac{\kappa}{4}, \quad k^{\Delta} k_{\Omega}^{\Sigma}=k_{\Omega}^{\Delta} .
$$

In this section we suppose that the two spin-3/2 gauge fields couple to gravity. It implies that the matrix of coefficients $k^{\Delta} \Omega$ is of maximal rank. In this case the above constraint is solved if and only if $k^{\Delta} \Omega=\delta_{\Omega}^{\Delta}$. We will be more general and not do this assumption in subsection 3.4 where the number of spin-3/2 gauge fields can remain arbitrary. The solution is given by

$$
\begin{aligned}
b_{2}= & \frac{\kappa^{2}}{4} \xi^{\star \alpha} \xi_{\mu}\left[h_{\alpha \nu} \partial^{[\mu} \xi^{\nu]}-h^{\mu \nu} \partial_{[\alpha} \xi_{\nu]}-\partial^{[\mu} h^{\nu]}{ }_{\alpha} \xi_{\nu}\right]+\frac{\kappa^{2}}{8} \xi_{\mu}^{\star} \xi_{\nu} \bar{\zeta}^{\Delta} \gamma^{[\mu} \psi_{\Delta}^{\nu]} \\
& +\frac{\kappa^{2}}{4} \zeta_{\Delta}^{\star} \gamma_{\mu \nu} \zeta^{\Delta}\left(\frac{1}{2} h^{\mu}{ }_{\sigma} \partial^{[\nu} \xi^{\sigma]}+\xi_{\sigma} \partial^{[\sigma} h^{\nu] \mu}\right)+\frac{\kappa^{2}}{32}\left(\zeta_{\Delta}^{\star} \gamma^{\mu \nu} \zeta^{\Delta}\right)\left(\bar{\zeta}_{\Omega} \gamma_{\mu} \psi_{\nu}^{\Omega}\right) .
\end{aligned}
$$

It encodes the gauge algebra to second order in the deformation. From now on there are 3 independent infinitesimal deformation parameters: $\kappa, y$ and $\beta$. The pure $\mathcal{N}_{4}=2$ supergravity theory without cosmological constant possesses a single dimensionful constant, namely the Newton constant, therefore we must expect that the equations (2.13) and (2.12) will impose constraints on the remaining constants, so that $\beta$ and $y$, say, will depend on $\kappa$. Let us proceed with equation (2.13) and consider, therein, all the terms that have the schematic structure " $A^{\star} F \xi \xi " \in H(\gamma)$ and therefore represent an obstruction to finding $b_{1}$, the deformations of the gauge transformation at second order in perturbation. We will give later - in (3.80) where $\beta$ is called $\alpha_{1}$ - the details of this computation when the background is $A d S_{4}$ and with an arbitrary number of Rarita-Schwinger and Maxwell gauge fields. It turns out that the only way to set to zero the coefficient in front of this obstruction is by imposing the relation

$$
\beta=\kappa .
$$

For what concerns the analysis of the terms of the schematic form $A^{\star} F \zeta \zeta \in H(\gamma)$ - we defer the treatment to (3.90), in $A d S_{4}$ background and for an arbitrary number $\mathcal{N}_{4}$ of Rarita-Schwinger gauge fields and $n_{v}$ Maxwell fields - , we have to impose

$$
y^{2}=\frac{\kappa^{2}}{32}
$$


in order to cancel the coefficient in front of the obstruction. This leaves us with only one deformation parameter $\kappa$. Let us show that we reproduced the pure $\mathcal{N}_{4}=2$ supergravity theory of [19].

Analysis of the deformation. Omitting the terms quartic in the spinors, the Lagrangian density $\mathcal{L}$ in [19] is given by

$$
\begin{gathered}
\mathcal{L}=\frac{\sqrt{-g}}{\kappa^{2}}\left[L^{\mathrm{EH}}-\frac{\kappa^{2}}{2} \bar{\psi}_{\mu}^{\Delta} \gamma^{\mu \nu \rho} \mathcal{D}_{\nu} \psi_{\rho}^{\Omega} \delta_{\Delta \Omega}-\frac{\kappa^{2}}{4} g^{\mu \rho} g^{\nu \sigma} F_{\mu \nu} F_{\rho \sigma}\right. \\
\left. \pm \frac{\kappa^{3}}{4 \sqrt{2}} \psi_{\mu}^{\Delta}\left(F^{\mu \nu} \mathbb{1}-i \gamma^{5}(* F)^{\mu \nu}\right) \psi_{\nu}^{\Omega} \epsilon_{\Delta \Omega}\right]
\end{gathered}
$$

where $\mathcal{D}_{\mu} \psi_{\nu}=\partial_{\mu} \psi_{\nu}+\frac{1}{4} \omega_{\mu}^{a b} \gamma_{a b} \psi_{\nu}$ is the Lorentz-covariant derivative on spinors. Since we study the deformations of a free theory around flat spacetime, we have ${ }^{4}$

$$
\begin{aligned}
g_{\mu \nu} & \equiv e^{a}{ }_{\mu} e_{a \nu}=\eta_{\mu \nu}+\kappa h_{\mu \nu}, & g^{\mu \nu} & =\eta^{\mu \nu}-\kappa h^{\mu \nu}+\mathcal{O}\left(h^{2}\right), \\
e^{a}{ }_{\mu} & =\delta_{\mu}^{a}+\frac{\kappa}{2} h_{\mu}^{a}, \quad e_{a}{ }^{\mu}=\delta_{a}^{\mu}-\frac{\kappa}{2} h_{a}{ }^{\mu}+\mathcal{O}\left(h^{2}\right), & e & \equiv \sqrt{-g}=1+\frac{\kappa}{2} h+\mathcal{O}\left(h^{2}\right) .
\end{aligned}
$$

Let us show that the cubic vertices (3.22) that we have derived are the cubic part of the $\mathcal{N}_{4}=2$ supergravity Lagrangian density $\mathcal{L}$. Firstly, the last term in (3.28) is directly given by $a_{0}^{(4)}$ of (3.17). Secondly, the expansion to third order of the third term in (3.28) is given by $a_{0}^{G 1}$. Finally, the cubic expansion of the second term in (3.28) correctly gives $a_{0}^{(2-7)}$ in (3.12). Actually, the first term of (3.12) gives the cubic part of $-\frac{1}{4} \bar{\psi}_{\mu}^{\Delta} \delta_{a}^{\mu} \delta_{b}^{\nu} \delta_{c}^{\rho} \gamma^{a b c} \omega_{\nu}^{p q} \sigma_{p q} \psi_{\rho}^{\Omega} \delta_{\Delta \Omega}$, while the others represent the cubic part of $e \bar{\psi}_{\mu}^{\Delta} \gamma^{a b c} \partial_{\nu} \psi_{\rho}^{\Omega} e_{a}{ }^{\mu} e_{b}{ }^{\nu} e_{c}{ }^{\rho} \delta_{\Delta \Omega}$.

We recall that the parts of the susy gauge transformations of the $\mathcal{N}_{4}=2$ supergravity theory that are linear in the fields are given by

$$
\begin{aligned}
& \delta_{\epsilon}^{(1)} A_{\mu} \propto \bar{\epsilon}^{\Delta} \psi_{\mu}^{\Omega} \epsilon_{\Delta \Omega} \\
& \delta_{\epsilon}^{(1)} e^{a}{ }_{\mu} \propto \bar{\epsilon}^{\Delta} \gamma^{a} \psi_{\mu}^{\Omega} \delta_{\Delta \Omega} \\
& \delta_{\epsilon}^{(1)} \psi_{\mu}^{\Delta} \propto \mathcal{D}_{\mu} \epsilon^{\Delta}+\epsilon^{\Delta \Omega}\left(F_{\mu \nu} \gamma^{\nu}+i(* F)_{\mu \nu} \gamma^{\nu} \gamma^{5}\right) \epsilon_{\Omega}
\end{aligned}
$$

The susy transformations of $A_{\mu}$ and $h_{\mu \nu}$ are directly given by $a_{1}^{(2)}$ and $a_{1}^{(4)}$ in (3.4) and (3.5). By use of (A.5) and (A.9) one can rewrite the last part of the susy gauge transformation of the gravitini as in $\tilde{a}_{1}^{(4)}(3.14)$, the first part being written in the second term of $a_{1}^{(7)}$ (see (3.7)) because $\stackrel{(1)}{\omega}_{\mu} \alpha \beta=-\partial^{[\alpha} h^{\beta]}{ }_{\mu}$ is the linearised spin connection in the Lorentz gauge where the antisymmetric part of the vierbein components is zero.

The rest of the gauge transformations is not written in [19] because they are just the transformations under diffeomorphisms. It should also be noted that, without cosmological constant, there is no deformation of the gauge transformations involving the U(1) parameter. In other words, the gravitini remain uncharged under U(1). The consistent minimal coupling of the gravitini to electromagnetism is the subject of the next subsection.

\footnotetext{
${ }^{4}$ The symbol $\kappa$ is chosen so that it corresponds to the dimensionful constant (related to the Newton constant by $\left.\kappa^{2}=\frac{32 \pi G}{c^{4}}\right)$ that appears in the perturbative expansion of the metric.
} 


\subsection{Obstruction to the $\mathrm{U}(1)$ minimal coupling}

This subsection is devoted to the obstruction coming from the resolution of (2.9) with source term $a_{1}^{(5)}(3.6)$. Acting with the Koszul-Tate differential on $a_{1}^{(5)}$ yields

$$
\delta a_{1}^{(5)}=\partial_{\mu} j^{\mu}-\gamma\left(\frac{1}{2} k_{\Delta \Omega}^{(5)} \bar{\psi}_{\rho}^{\Delta} \gamma^{\mu \nu \rho} \psi_{\nu}^{\Omega} A_{\mu}\right)+\underbrace{\frac{1}{2} k_{\Delta \Omega}^{(5)} F_{\mu \nu} \bar{\psi}_{\rho}^{\Delta} \gamma^{\mu \nu \rho} \zeta^{\Omega}}_{\text {Obstruction }} .
$$

The last term is an obstruction to finding a cubic vertex $a_{0}^{(5)}$ in the resolution of (2.9), because it cannot be written as a $\gamma$-exact term modulo $\partial_{\mu} j^{\mu}$, for both the antisymmetric and the symmetric parts of $k_{\Delta \Omega}^{(5)}$. This can be seen by taking the Euler-Lagrange derivative of it with respect with $A_{\mu}$ and checking that it gives an element in $H(\gamma)$. The only way to cancel this obstruction without adding extra deformation is by choosing $k_{\Delta \Omega}^{(5)}$ to be identically zero. Treating the resolution of $a_{2}^{(5)}$ together with another $a_{2}$ or adding an $\tilde{a}_{1}^{(5)} \in H(\gamma)$ does not help because the Koszul-Tate differential $\delta$ acting on them gives at least two derivatives while the obstruction only has one derivative.

The nontrivial way to cancel this obstruction, thereby saving the minimal coupling in a Poincaré-invariant deformation procedure around flat background, is by introducing an infinitesimal deformation that is linear in the dynamical fields. The only such Poincaréinvariant deformation is

$$
W_{1}^{\text {lin. }}=\int d^{4} x\left(-2 \tilde{\lambda}^{2} h\right) .
$$

It is a first order deformation of the master equation because the BRST differential acting on $h=\eta^{\mu \nu} h_{\mu \nu}$ gives a total derivative [11]. It is easy to see that there is no other Poincaréinvariant, infinitesimal deformation that is linear in the fields of the spectrum at hand. If one accepts to break Poincaré symmetry by explicitly introducing Cartesian coordinates $x^{\mu}$ dependence into the higher-order deformations, the above deformation can be seen to trigger a change of background, leading from flat to $(A) d S_{4}$ background. ${ }^{5}$ Note that, with the first-order infinitesimal deformation (3.35), linear in the weak field, the degree of polynomiality of the deformation is not related to the degree of perturbation by a shift of 2. For example, around flat space, with the infinitesimal cosmological constant deformation (3.35) being introduced, cubic vertices may appear at second order in deformation, instead of appearing at first order.

Sticking to Poincaré invariance for the moment and in order for the deformation to be consistent at second order in the presence of real Rarita-Schwinger gauge fields, one also needs [21] to add a mass term for the massless spin- $\frac{3}{2}$ fields, which translates into the following additional infinitesimal deformations, this time quadratic in the fields:

$$
\begin{aligned}
& a_{1}^{m}=-\frac{m}{2} \psi_{\Delta}^{\star \mu} \gamma_{\mu} \zeta^{\Delta}, \\
& a_{0}^{m}=-\frac{m}{2} \bar{\psi}^{\mu \Delta} \gamma_{\mu \nu} \psi_{\Delta}^{\nu},
\end{aligned}
$$

\footnotetext{
${ }^{5}$ The $A d S_{4}$ background permits unitary representation for the spinors. One way to accommodate the $d S_{4}$ background is by changing the reality condition on the spinors, but as explained in [28], in the interacting theory the sign in front of the Maxwell action will have to flip in order to keep the vector fields real, leading to a classical ghost. Alternatives to supergravity models in de Sitter background can be found in [29] and refs. therein.
} 
the mass being related to the cosmological constant that has to be negative for Majorana spinors, as was re-derived in [21], consistently with the original finding of [22].

As explained in the Introduction, instead of proceeding with the study of the Poincaréinvariant deformations around flat space that include the new cosmological pieces given above, a procedure that can certainly be pursued but has the aesthetic inconvenience of having first-order deformations mixing up expressions of first, second and third polynomial orders in the weak fields, the strategy we prefer (and that leads to the same results) is to start the deformation procedure from the solution of the classical master equation around $\mathrm{AdS}_{4}$ with finite (negative) cosmological constant, asking for $\mathrm{SO}(2,3)$-invariant infinitesimal deformations. Since in all cases we see that we have to introduce gravity and the diffeomorphism algebra for consistency of the couplings, thereby leading to a background independent end result, the advantage with the latter approach is that the minimal coupling terms will appear at first order in deformation around $A d S_{4}$, instead of appearing at second order in deformation around flat background. The solution of the classical master equation associated with the free theory in $A d S_{4}$ background is given in (2.24). The flat limit $\lambda \longrightarrow 0$ of this functional is smooth and reproduces the solution of the master equation (2.15) for the free theory in Minkowski spacetime.

\subsection{Deformations around AdS background}

In this section, we therefore start from the functional (2.24) and compute its most general, infinitesimal cubic deformations that preserve the $\mathrm{SO}(2,3)$ symmetries of the $A d S_{4}$ background. As in the previous sections and following the general method of [24], we first classify all the possible deformations that deform the gauge algebra, and for each of them, we determine whether they can be lifted to give deformations of the gauge transformations, and possibly a cubic vertex. Along that way, we also determine the deformations that do not alter the abelian gauge algebra of the free theory but that nevertheless deform the gauge transformations laws. The analyses of this section are performed for $\mathcal{N}_{4}$ arbitrary, however, due to the spectrum of fields that we allow, consistent theories to all orders will only be obtained for the cases where $\mathcal{N}_{4}<4$. Some of the constraints we obtain (those that come from (2.14)) remain unchanged whenever one introduces extra matter fields in the form of scalars and Majorana spinors, whereas other constraints will change when additional matter fields will be introduced.

We start with

$$
s W_{1}=0,
$$

set $W_{1}=\int d^{4} x \sqrt{-\bar{g}}\left(a_{0}+a_{1}+a_{2}\right)$ and expand the above equation with respect to the antighost number:

$$
\begin{aligned}
\delta a_{1}+\gamma a_{0} & =\partial_{\mu} j_{0}^{\mu}=\sqrt{-\bar{g}} \nabla_{\mu} \tilde{j}_{0}^{\mu}, \\
\delta a_{2}+\gamma a_{1} & =\partial_{\mu} j_{1}^{\mu}=\sqrt{-\bar{g}} \nabla_{\mu} \tilde{j}_{1}^{\mu}, \\
\gamma a_{2} & =0 .
\end{aligned}
$$

We search for solutions with non-trivial $a_{2}$ and therefore have to classify the possible cubic $a_{2}$ 's belonging to the cohomological groups $H(\gamma)$ in pureghost number 2 and form degree 
zero. The classification of these terms is straigthforward and has already been presented in the flat space case in (3.1)-(3.3). All we have to do is to covariantise these candidates to the $A d S_{4}$ background, adding mass-like terms when they are allowed, and adding the YangMills deformation term $\frac{1}{2} \mathcal{C}_{a}^{\star} f_{b c}^{a} \mathcal{C}^{b} \mathcal{C}^{c}$ since we now allow for an arbitrary number $n_{v}$ of free abelian vector fields. We present only those $a_{2}$ 's that lead to corresponding $a_{1}$ and $a_{0}$, upon solving (3.39)-(3.41), without encountering any obstruction. The final result reads $a_{2}=\kappa a_{2}^{\mathrm{EH}}+\alpha_{3 / 2} a_{2}^{\text {susy }}+y a_{2}^{c c}+g_{\mathrm{YM}} a_{2}^{\mathrm{YM}}$ and generates the algebras of diffeomorphisms, local supersymmetries with "central charges" in $A d S_{4}$, and Yang-Mills, respectively. The constant parameters $\kappa, \alpha_{3 / 2}, y$ and $g_{\mathrm{YM}}$ are infinitesimal deformation parameters that we choose in this paper not to absorb in the corresponding structure constants. We present the results of this classification in various paragraphs, with titles referring to the infinitesimal deformations of the gauge algebra for the various linear combinations of $a_{2}$ 's.

Diffeomorphisms. The diffeomorphism algebra deformation, around $A d S_{4}$, is obtained from the following candidate:

$$
a_{2}^{\mathrm{EH}}=\xi^{\star \mu} \xi^{\nu} \nabla_{[\mu} \xi_{\nu]}
$$

This candidate can be lifted to a deformation of the gauge transformations through

$$
a_{1}^{\mathrm{EH}}=h^{\star \mu \nu}\left[\nabla_{\mu} \xi^{\sigma} h_{\nu \sigma}-\xi^{\sigma}\left(\nabla_{\mu} h_{\nu \sigma}-\nabla_{\sigma} h_{\mu \nu}\right)\right] .
$$

Of course, as in [11] but this time in the $A d S_{4}$ background, one can make a trivial redefinition of $a_{2}^{\mathrm{EH}}$ in order to read in the corresponding $a_{1}$ the transformations of $h_{\mu \nu}$ given by its Lie derivative along $\xi^{\mu}$, the gauge parameters associated with infinitesimal diffeomorphisms. Then we can lift $a_{1}^{\mathrm{EH}}$ to get the cubic vertex of the Einstein-Hilbert action with negative cosmological constant around $A d S_{4}$ in such a way that the cosmological constant terms appear explicitly as the cubic part of $\sqrt{-g}$. The latter cubic terms can of course appear with different relative coefficients compared to the mere expansion of $\sqrt{-g}$ to third order around $A d S_{4}$, the reason being the ambiguity in the cubic terms with two $A d S_{4}$-covariant derivatives, once one integrates by parts. We have fixed this freedom in integration by parts by requiring that the undifferentiated cubic terms exactly produce the cubic terms in the expansion of $\sqrt{-g}$ around $A d S_{4}$. In this way, we obtain the following cubic vertex:

$$
\begin{aligned}
a_{0}^{\mathrm{EH}}= & \frac{1}{2} h^{\mu \nu} \nabla_{\mu} h_{\rho \sigma} \nabla_{\nu} h^{\rho \sigma}-\frac{1}{2} h^{\mu \nu} \nabla_{\mu} h \nabla_{\nu} h+h^{\mu \nu} \nabla_{\mu} h \nabla^{\sigma} h_{\sigma \nu}+h^{\mu \nu} \nabla_{\mu} h_{\nu \sigma} \nabla^{\sigma} h \\
& -h^{\mu \nu} \nabla_{\sigma} h_{\mu \nu} \nabla^{\sigma} h+\frac{1}{4} h \nabla_{\mu} h \nabla^{\mu} h+h^{\mu \nu} \nabla_{\sigma} h_{\mu \nu} \nabla_{\rho} h^{\rho \sigma}-\frac{1}{2} h \nabla_{\mu} h \nabla_{\nu} h^{\mu \nu} \\
& -2 h^{\mu}{ }_{\nu} \nabla_{\mu} h_{\rho \sigma} \nabla^{\rho} h^{\sigma \nu}-h^{\mu}{ }_{\nu} \nabla_{\sigma} h_{\rho \mu} \nabla^{\rho} h^{\sigma \nu}+h^{\mu}{ }_{\nu} \nabla_{\sigma} h_{\rho \mu} \nabla^{\sigma} h^{\rho \nu}+\frac{1}{2} h \nabla_{\sigma} h_{\mu \nu} \nabla^{\mu} h^{\nu \sigma} \\
& -\frac{1}{4} h \nabla_{\sigma} h_{\mu \nu} \nabla^{\sigma} h^{\mu \nu}+\lambda^{2}\left(\frac{1}{2} h h h+4 h_{\mu}{ }^{\nu} h_{\nu}{ }^{\rho} h_{\rho}{ }^{\mu}-3 h h_{\mu \nu} h^{\mu \nu}\right)
\end{aligned}
$$


Supersymmetry algebra. The next gauge-algebra deformation candidate that can be lifted all the way to a deformation of the gauge transformations and to a cubic vertex reads

$$
\begin{aligned}
\alpha_{3 / 2} a_{2}^{\text {susy }}+y a_{2}^{c c-\Lambda}= & \alpha_{3 / 2}\left(\frac{1}{4} \xi^{\star \mu} \bar{\zeta}_{\Delta} \gamma_{\mu} \zeta_{\Omega} k^{\Delta \Omega}+\zeta_{\Delta}^{\star} \gamma^{\mu \nu} \zeta_{\Omega} \nabla_{[\mu} \xi_{\nu]} k^{\Delta \Omega}-2 \lambda \zeta_{\Delta}^{\star} \gamma^{\mu} \zeta_{\Omega} \xi_{\mu} k^{\Delta \Omega}\right) \\
& +y\left(\mathcal{C}_{a}^{\star} \bar{\zeta}^{[\Delta} \zeta^{\Omega]} t^{a}{ }_{\Delta \Omega}-2 \lambda \zeta^{\star}{ }_{\Delta} \zeta_{\Omega} \mathcal{C}^{a} t_{a}{ }^{\Delta \Omega}\right), \quad t^{a}{ }_{\Delta \Omega}=-t^{a}{ }_{\Omega \Delta}
\end{aligned}
$$

The coefficient $-2 \lambda$ in front of the third term above is fixed by requiring that the $a_{2}$ candidate can be lifted to an $a_{1}$. All other new - as compared to the flat space case studied previously - linear relations between the coefficients of the $a_{2}$ 's appear when lifting the $a_{1}$ to $a_{0}$. We can lift that linear combination to obtain the following infinitesimal gauge-transformation deformation $a_{1}$ :

$$
\begin{aligned}
\alpha_{3 / 2} a_{1}^{\text {susy }}+y a_{1}^{c c-\Lambda}=\alpha_{3 / 2} & {\left[-h^{\star \mu \nu} \bar{\psi}_{\mu \Delta} \gamma_{\nu} \zeta_{\Omega} k^{\Delta \Omega}+\psi_{\Delta}^{\star \rho} \gamma_{\mu \nu} \psi_{\Omega \rho} \nabla^{[\mu} \xi^{\nu]} k^{\Delta \Omega}\right.} \\
& \left.\quad-\psi_{\Delta}^{\star \rho} \gamma^{\mu \nu} \zeta_{\Omega} \nabla_{[\mu} h_{\nu] \rho} k^{\Delta \Omega}+\lambda \psi_{\Delta}^{\star \mu} \gamma^{\nu} \zeta_{\Omega} h_{\mu \nu} k^{\Delta \Omega}-2 \lambda \psi_{\Delta}^{\star \mu} \gamma_{\nu} \psi_{\Omega \mu} \xi^{\nu} k^{\Delta \Omega}\right] \\
+y & {\left[-2 A_{a}^{\star \mu} \bar{\psi}_{\mu}^{\Delta} \zeta^{\Omega} t^{a}{ }_{\Delta \Omega}-2 \lambda \psi_{\Delta}^{\star \mu}\left(\psi_{\mu \Omega} \mathcal{C}^{a}-\zeta_{\Omega} A_{\mu}^{a}\right) t_{a}{ }^{\Delta \Omega}\right] . }
\end{aligned}
$$

This term can be lifted to get a vertex $a_{0}$ only provided we add to it an appropriate cocycle $\tilde{a}_{1}$ of $H(\gamma)$, that is to say, an appropriate solution to the homogeneous equation $\gamma \tilde{a}_{1}=0$ :

$$
\alpha_{3 / 2} \tilde{a}_{1}^{G 3 / 2}+y \tilde{a}_{1}^{c c}=-8 \alpha_{3 / 2} \psi_{\Delta}^{\star \mu} \Psi_{\mu \nu \Omega} \xi^{\nu} k^{\Delta \Omega}-\frac{1}{2} y \psi_{\mu \Delta}^{\star} F_{\rho \sigma}^{a} \gamma^{\rho \sigma} \gamma^{\mu} \zeta_{\Omega} t_{a}{ }^{\Delta \Omega} .
$$

Only after the addition of this term to (3.46) can we get from the equation (3.39) a cubic vertex $a_{0}$ in the form

$$
\begin{aligned}
\alpha_{3 / 2} a_{0}^{\text {susy }}+y a_{0}^{c c}=\alpha_{3 / 2} & \left(\bar{\psi}_{\mu}^{\Delta} \gamma^{\mu \nu \rho} \sigma^{\alpha \beta} \nabla_{[\alpha} h_{\beta] \nu} \psi_{\rho}^{\Omega} k_{\Delta \Omega}\right. \\
& -h \bar{\psi}_{\mu}^{\Delta} \gamma^{\mu \nu \rho} \nabla_{\nu} \psi_{\rho}^{\Omega} k_{\Delta \Omega}+h_{\alpha \beta} \bar{\psi}^{\alpha \Delta} \gamma^{\beta \nu \rho} \nabla_{\nu} \psi_{\rho}^{\Omega} k_{\Delta \Omega}+h_{\alpha \beta} \bar{\psi}_{\mu}^{\Delta} \gamma^{\mu \alpha \nu} \nabla^{\beta} \psi_{\rho}^{\Omega} k_{\Delta \Omega} \\
& \left.+h_{\alpha \beta} \bar{\psi}_{\mu}^{\Delta} \gamma^{\mu \nu \alpha} \nabla_{\nu} \psi^{\beta \Omega} k_{\Delta \Omega}+\lambda h \bar{\psi}_{\mu}^{\Delta} \gamma^{\mu \nu} \psi_{\nu}^{\Omega} k_{\Delta \Omega}-2 \lambda h_{\alpha \beta} \bar{\psi}^{\alpha \Delta} \gamma^{\beta \nu} \psi_{\nu}^{\Omega} k_{\Delta \Omega}\right) \\
& +y\left(\bar{\psi}_{\mu}^{\Delta}\left(F_{a}^{\mu \nu}-i \gamma^{5}\left(* F_{a}\right)^{\mu \nu}\right) \psi_{\nu}^{\Omega} t^{a}{ }_{\Delta \Omega}-\lambda \bar{\psi}_{\Delta \rho} \gamma^{\mu \nu \rho} \psi_{\Omega \nu} A_{\mu}^{a} t_{a}{ }^{\Delta \Omega}\right) .
\end{aligned}
$$

Yang-Mills algebra. The infinitesimal deformation corresponding to the Yang-Mills interactions, as obtained from the cohomological procedure [7], is well-known [30]. We recall it here for completeness:

$$
a_{2}^{\mathrm{YM}}=\frac{1}{2} \mathcal{C}_{c}^{\star} f^{c}{ }_{a b} \mathcal{C}^{a} \mathcal{C}^{b},
$$

where $f_{a b}^{c}=-f_{b a}^{c}$. After solving the first order descent equations (3.39)-(3.41), one obtains

$$
\begin{aligned}
& a_{1}^{\mathrm{YM}}=A_{c}^{\star \mu} A_{\mu}^{a} \mathcal{C}^{b} f^{c}{ }_{a b}, \\
& a_{0}^{\mathrm{YM}}=-\frac{1}{2} F_{c}^{\mu \nu} A_{\mu}^{a} A_{\nu}^{b} f^{c}{ }_{a b},
\end{aligned}
$$

provided that

$$
f_{a b c}:=f_{b c}^{d} \delta_{a d}=f_{[a b c]} .
$$


Total first order deformation of the gauge algebra. Summarising the results obtained so far, the total infinitesimal deformation of the gauge algebra that can be lifted up to a cubic vertex $a_{0}$ is given by

$$
a_{2}=\kappa a_{2}^{\mathrm{EH}}+\alpha_{3 / 2} a_{2}^{\text {susy }}+y a_{2}^{c c-\Lambda}+g_{\mathrm{YM}} a_{2}^{\mathrm{YM}} .
$$

It depends on the four independent deformation parameters $\kappa, \alpha_{3 / 2}, y$ and $g_{\mathrm{YM}}$. We will see in the rest of this section how the existence of a second-order deformation will relate these four infinitesimal constants.

Before turning to the second-order deformations, we want to classify the first-order deformations of the gauge transformations that do not modify the gauge algebra. The corresponding descent equations are

$$
\begin{aligned}
\delta \hat{a}_{1}+\gamma a_{0} & =\sqrt{-\bar{g}} \nabla_{\mu} j_{0}^{\mu}, \\
\gamma \hat{a}_{1} & =0 .
\end{aligned}
$$

We present the classification of the possible terms in the following paragraphs.

Coupling of the vector fields to gravity. The following cocycle of $H(\gamma)$ at antifield number 2 ,

$$
\hat{a}_{1}^{G 1}=-k^{a b} A_{a}^{\star \mu} F_{b \mu \nu} \xi^{\nu}
$$

can be lifted to the cubic vertex

$$
a_{0}^{G 1}=-\frac{1}{8} h F_{a}^{\mu \nu} F_{b \mu \nu} k^{a b}-\frac{1}{2} F_{a}^{\mu \sigma} F_{b \sigma}{ }^{\nu} h_{\mu \nu} k^{a b}, \quad k_{a b}=k_{b a} .
$$

Coupling of the spin- $\frac{1}{2}$ field to gravity. The cocycle

$$
\hat{a}_{1}^{G 1 / 2}=\chi^{\star} \gamma^{\mu \nu} \chi \nabla_{[\mu} \xi_{\nu]}+4 \chi^{\star} \nabla_{\mu} \chi \xi^{\mu}
$$

can be lifted through (3.54) to yield the cubic vertex

$$
a_{0}^{G 1 / 2}=\bar{\chi} \gamma^{\mu} \nabla^{\nu} \chi h_{\mu \nu}-\bar{\chi} \gamma^{\mu} \nabla_{\mu} \chi h .
$$

Interaction between the spin- $\frac{3}{2}$, the spin-1 and the spin- $\frac{1}{2}$ fields. Finally, the following cocycle of $H(\gamma)$,

$$
\hat{a}_{1}^{\text {int }}=\frac{1}{2} k_{a \Delta} \chi^{\star} F_{\mu \nu}^{a} \gamma^{\mu \nu} \zeta^{\Delta}+k^{a \Delta} A_{a}^{\star \mu} \bar{\zeta}_{\Delta} \gamma_{\mu} \chi,
$$

can be lifted in (3.54) to produce the cubic interaction ${ }^{6}$

$$
a_{0}^{\mathrm{int}}=\frac{1}{2} k_{a \Delta} \bar{\chi} \gamma^{\lambda} \gamma^{\mu \nu} F_{\mu \nu}^{a} \psi_{\lambda}^{\Delta}
$$

\footnotetext{
${ }^{6}$ We could have allowed a nonzero mass term for the Majorana spin- $\frac{1}{2}$ field, since anyway it has no gauge invariance. In the case of a non-zero mass, however, it is easy to see that there is an obstruction to the existence of $a_{0}^{\text {int }}$. Since, on the other hand, second-order considerations show that this vertex is necessary for the consistency of a theory that requires a Majorana spin- $\frac{1}{2}$ field, we find that the mass of the Majorana spin- $\frac{1}{2}$ field must vanish.
} 
Total first order deformation of the gauge transformations. To summarise, the first-order infinitesimal deformations of the free theory (2.24), in the sector $a_{1}$ corresponding to the deformations of the gauge transformations, is given by the linear combination

$a_{1}=\kappa a_{1}^{\mathrm{EH}}+\alpha_{3 / 2}\left(a_{1}^{\mathrm{susy}}+\tilde{a}_{1}^{G 3 / 2}\right)+y\left(a_{1}^{c c-\Lambda}+\tilde{a}_{1}^{c c}\right)+g_{\mathrm{YM}} a_{1}^{\mathrm{YM}}+\alpha_{1} \hat{a}_{1}^{G 1}+\alpha_{1 / 2} \hat{a}_{1}^{G 1 / 2}+\omega \hat{a}_{1}^{\mathrm{int}}$,

which depends on seven independent deformation parameters: $\kappa, \alpha_{3 / 2}, y, g_{\mathrm{YM}}, \alpha_{1}, \alpha_{1 / 2}$ and $\omega$.

\subsection{General and specific quadratic constraints}

The goal of this subsection is to solve the second order master equation

$$
s W_{2}=-\frac{1}{2}\left(W_{1}, W_{1}\right) .
$$

We expand $W_{2}$ in antifield number, $W_{2}=\int d^{4} x \sqrt{-\bar{g}}\left(b_{0}+b_{1}+b_{2}\right)$, and insert the expression in the above equation that produces the following descent of equations:

$$
\begin{aligned}
\delta b_{1}+\gamma b_{0} & =-\sqrt{-\bar{g}}\left(a_{1}, a_{0}\right)+\partial_{\mu} t_{0}^{\mu}, \\
\delta b_{2}+\gamma b_{1} & =-\frac{1}{2} \sqrt{-\bar{g}}\left(a_{1}, a_{1}\right)-\sqrt{-\bar{g}}\left(a_{2}, a_{1}\right)+\partial_{\mu} t_{1}^{\mu}, \\
\gamma b_{2} & =-\frac{1}{2} \sqrt{-\bar{g}}\left(a_{2}, a_{2}\right)+\partial_{\mu} t_{2}^{\mu} .
\end{aligned}
$$

Descent equation of maximal antighost number. This paragraph is devoted to the resolution of equation (3.65). The latter resolution is straightforward, although rather lengthy, therefore we only list the obstructions encountered in the resolution of (3.65). The obstructions are elements of $H(\gamma)$ that remain non-trivial in $H(\gamma \mid d)$. In order to remove all the obstructions we have to impose some constraints between the deformation parameters and some constraints on the normalized coefficients. In the computation of (3.65), other obstructions appear with independent structures, but multiplied by the same coefficients as those appearing below in (3.66)-(3.69). Therefore, setting these coefficients to zero kills several different obstructions simultaneously. The minimal set of obstructions that bring the relevant coefficients to be set to zero is:

$$
\begin{aligned}
& \mathcal{O}_{1}=\alpha_{3 / 2}\left(\alpha_{3 / 2} k^{\Delta}{ }_{\Sigma} k_{\Omega}^{\Sigma}-\frac{\kappa}{4} k^{\Delta}{ }_{\Omega}\right) \xi^{\star \mu} \nabla_{[\mu} \xi_{\nu]} \bar{\zeta}_{\Delta} \gamma^{\nu} \zeta^{\Omega}, \\
& \mathcal{O}_{2}=\lambda\left(\alpha_{3 / 2}^{2} 2 k_{\Delta \Omega} k_{\Lambda \Gamma}-2 y^{2} t_{a \Delta \Gamma} t_{\Omega \Lambda}^{a}\right)\left(\zeta^{\star \Delta} \zeta^{\Gamma}\right)\left(\bar{\zeta}^{[\Omega} \zeta^{\Lambda]}\right), \\
& \mathcal{O}_{3}=\lambda y\left(2 \lambda y 2\left(t_{a}\right)^{\Delta}{ }_{\Sigma}\left(t_{b}\right)^{\Sigma}{ }_{\Omega}+g_{\mathrm{YM}} f_{a b}^{c}\left(t_{c}\right)^{\Delta}{ }_{\Omega}\right) \zeta_{\Delta}^{\star} \zeta^{\Omega} C^{[a} C^{b]}, \\
& \mathcal{O}_{4}=-g_{\mathrm{YM}}^{2}\left(f_{a b}^{c} f_{d e}^{a}{ }_{d e}\right) C_{c}^{\star} C^{[b} C^{d} C^{e]} .
\end{aligned}
$$

For these obstructions to vanish, a necessary condition we have to impose are the following relations on the deformation parameters:

$$
\begin{aligned}
\alpha_{3 / 2} & =\frac{\kappa}{4}, \\
y^{2} & =\frac{\kappa^{2}}{32}, \\
g_{\mathrm{YM}} & =-2 \lambda y .
\end{aligned}
$$


There thus remains only one free deformation parameter in the expression of $a_{2}$, in (3.53), leaving us with four deformation parameters in total, taking into account those appearing in (3.61). Then, in order for the four obstructions above to be identically zero we also have to impose constraints on the normalized coefficients involved in the definition of the $a_{2}$ 's. First of all we must have

$$
k^{\Delta} k_{\Omega}^{\Sigma}=k^{\Delta} \Omega .
$$

If $k^{\Delta} \Omega$ is of maximal rank, that is to say if we choose that all the gravitini are coupled to gravity, then this normalised matrix is invertible and the only solution to the above constraint is $k^{\Delta}{ }_{\Omega}=\delta_{\Omega}^{\Delta}$. However, we will also consider the case where the matrix with components $k^{\Delta} \Omega$ has the zero eigenvalue, with some multiplicity. Another constraint that appears from the vanishing of the obstruction (3.69) is the Jacobi identity

$$
f_{a[b}^{c} f_{d e]}^{a}=0,
$$

which tells us that $f_{a b}^{c}$ are the structure constants of a Lie algebra for which $\delta_{a b}$ is an invariant tensor. From the complete antisymmetry and reality of $f_{a b c}$, one recovers [30] that the real Lie algebra must be semi-simple and compact.

Then, if we see the coefficients $t_{a}{ }^{\Delta} \Omega$ as $n_{v}$ square $\mathcal{N}_{4} \times \mathcal{N}_{4}$ matrices, we have the constraint

$$
\left[t_{a}, t_{b}\right]^{\Delta}{ }_{\Omega}=f_{a b}^{c}\left(t_{c}\right)^{\Delta} \Omega
$$

that means that these matrices characterise a representation of dimension $\mathcal{N}$ of the compact Lie group defined by the structure constants $f_{a b}^{c}$. Finally, we have the completeness relation

$$
\left(t^{a}\right)_{\Delta \Omega}\left(t_{a}\right)^{\Gamma \Sigma}=2 k^{[\Gamma}{ }_{\Delta} k^{\Sigma]} \Omega
$$

that also comes as a constraint in order to remove the obstruction (3.67) that appear in solving (3.65). Once the above constraints are satisfied, we can solve (3.65) to obtain the deformation of the gauge algebra, to second order in deformation:

$$
\begin{aligned}
b_{2}= & \kappa^{2} b_{2}^{\mathrm{EH}}+\frac{\kappa^{2}}{4} k^{\Delta \Omega} \zeta_{\Delta}^{\star} \gamma_{\mu \nu} \zeta_{\Omega}\left(\frac{1}{2} h_{\sigma}^{\mu} \nabla^{[\nu} \xi^{\sigma]}+\xi_{\sigma} \nabla^{[\sigma} h^{\nu] \mu}\right) \\
& +\frac{\kappa^{2}}{8} k^{\Delta \Omega} \xi_{\mu}^{\star} \xi_{\nu} \bar{\zeta}_{\Delta} \gamma^{[\mu} \psi_{\Omega}^{\nu]}-\frac{\kappa^{2}}{32} k^{\Delta \Omega} k_{\Gamma \Sigma}\left(\zeta_{\Delta}^{\star} \gamma^{\mu \nu} \zeta_{\Omega}\right)\left(\bar{\zeta}^{\Gamma} \gamma_{\nu} \psi_{\mu}^{\Sigma}\right),
\end{aligned}
$$

up to trivial terms and up to solutions $\tilde{b}_{2}$ to the homogeneous equation $\gamma \tilde{b}_{2}=0$. In the above expression, $b_{2}^{\mathrm{EH}}$ is the second-order deformation of the diffeomorphism algebra:

$$
b_{2}^{\mathrm{EH}}=\frac{1}{4} \xi^{\star \alpha} \xi_{\mu}\left[h_{\alpha \nu} \nabla^{[\mu} \xi^{\nu]}-h^{\mu \nu} \nabla_{[\alpha} \xi_{\nu]}-\nabla^{[\mu} h^{\nu]}{ }_{\alpha} \xi_{\nu}\right],
$$

At this stage we can make two remarks on our preliminary results obtained in $A d S_{4}$ background:

(i) We can obtain the expression of the charge of the gravitini under the gauge group in terms of the Newton constant and the cosmological constant by reading off the coefficient in front of the minimal coupling terms in (3.48). Using (3.71), it is given by

$$
-\lambda y=\mp \frac{\lambda \kappa}{4 \sqrt{2}},
$$


where the ambiguity of sign translates the fact that the charge of the gravitini can have both signs;

(ii) Had we calculated the deformations around Minkowski spacetime, we would have found the minimal coupling of the gravitini to the gauge group at second order in deformation after the introduction of the first-order deformations (3.35)-(3.37), since around flat space the cosmological constant is an infinitesimal deformation parameter. Moreover, neither (3.67) nor (3.68) would have appeared as obstructions during the resolution of the equation (2.14) around flat spacetime, since the latter expressions are multiplied by $\lambda$. Nevertheless we also find the constraint $y^{2}=\frac{\kappa^{2}}{32}$, this time during the resolution of (2.13). Actually, perturbatively, we can recover all the deformations that one can obtain around Minkowski spacetime by taking the limit $\lambda \longrightarrow 0$ of the deformation around $A d S_{4}$ spacetime;

(iii) Independently of the matter content, the constraints (3.73)-(3.76) must hold for the existence of any consistent theory involving spin- 1 and spin- $3 / 2$ gauge fields.

Descent equation at antifield number 1. After having solved the equation (3.65) at antifield number 2 , with the expression of $b_{2}$ given above, we can plug the result into the equation (3.64) and solve for $b_{1}$, the deformation of the gauge algebra at second order in deformation. If obstructions arise, the vanishing of the coefficients of the obstructions will give extra quadratic constraints on the deformation parameters and structure constants, in particular on the constants $\alpha_{1}$ and $\alpha_{1 / 2}$ that appear in (3.64). The ambiguity $\hat{b}_{2}$ in the solution (3.77) will affect $b_{1}$ through on-shell closure terms. These terms are expected to arise as the supersymmetry gauge algebra only closes on-shell, when no auxiliary fields are introduced. On top of $\hat{b}_{2}$ terms with the general structure " $\psi^{\star} \psi^{\star} \zeta \zeta$ " we also find that terms of the schematic form " $\chi^{\star} A^{\star} \zeta \xi$ ", for example, must be introduced in order to find the $b_{1}$ terms, namely, the deformations of the gauge transformations at second order in the fields.

Determination of $\alpha_{1}$. We fix the value of the constant parameter $\alpha_{1}$ by inspection of the terms in (3.64) having the schematic form " $A^{\star} F \xi \xi$ ", belonging to the cohomological group $H(\gamma)$. These terms cannot come from $\delta b_{2}$ (because there is no $\mathcal{C}^{\star}$ in $b_{2}$ ) but can only come from $\left(\hat{a}_{1}^{G 1}, \hat{a}_{1}^{G 1}\right)$ and $\left(a_{2}^{\mathrm{EH}}, \hat{a}_{1}^{G 1}\right)$. Then, provided one adds the homogeneous piece $\left(\alpha_{1}\right)^{2} \tilde{b}_{2}^{G 1}=-\frac{\left(\alpha_{1}\right)^{2}}{2} \mathcal{C}_{a}^{\star} \xi^{\mu} \xi^{\nu} F_{\mu \nu}^{b} k_{c}^{a} k_{b}^{c}$ to $b_{2}$, the only obstruction that comes in the calculation of $-\frac{\left(\alpha_{1}\right)^{2}}{\sqrt{-\bar{g}}} \delta \tilde{b}_{2}^{G 1}-\frac{1}{2}\left(\hat{a}_{1}^{G 1}, \hat{a}_{1}^{G 1}\right)-\left(a_{2}^{\mathrm{EH}}, \hat{a}_{1}^{G 1}\right)+\nabla_{\mu} t_{1}^{\mu}$ is

$$
\mathcal{O}_{5}=\alpha_{1}\left(\kappa k_{b}^{a}-\alpha_{1} k_{c}^{a} k_{b}^{c}\right) A_{a}^{\star \sigma} \xi^{\nu} \nabla_{[\mu} \xi_{\nu]} F_{\sigma}^{b}{ }^{\mu}
$$

leading to the two constraints

$$
\alpha_{1}=\kappa, \quad k_{b}^{a}=k_{c}^{a} k_{b}^{c} .
$$

Provided the matrix with coefficients $k_{b}^{a}$ has maximal rank, meaning that all the spin-1 gauge field couple to gravity, the second constraint is solved by $k_{b}^{a}=\delta_{a}^{b}$, but otherwise, some vector fields can remain decoupled from gravity. 
Determination of $\alpha_{1 / 2}$. The determination of $\alpha_{1 / 2}$ is realized by an analysis of the terms " $\chi^{\star} \chi \nabla \xi \xi$ " that appear in the resolution of (3.64). These terms can only arise from $\left(\hat{a}_{1}^{G 1 / 2}, \hat{a}_{1}^{G 1 / 2}\right)$ and $\left(a_{2}^{\mathrm{EH}}, \hat{a}_{1}^{G 1 / 2}\right)$. Here, three obstruction appears:

$$
\begin{aligned}
& \mathcal{O}_{6}=4 \alpha_{1 / 2}\left(4 \alpha_{1 / 2}-\kappa\right) \chi^{\star} \nabla_{\mu} \chi \xi_{\nu} \nabla^{[\mu} \xi^{\nu]} \\
& \mathcal{O}_{7}=\alpha_{1 / 2}\left(4 \alpha_{1 / 2}-\kappa\right) \chi^{\star} \gamma^{\mu \alpha} \chi \bar{g}_{\beta \nu} \nabla_{[\mu} \xi_{\nu]} \nabla_{[\alpha} \xi_{\beta]}, \\
& \mathcal{O}_{8}=-\alpha_{1 / 2} \lambda^{2}\left(4 \alpha_{1 / 2}-\kappa\right) \chi^{\star} \gamma^{\mu \nu} \chi \xi_{\mu} \xi_{\nu}
\end{aligned}
$$

The coefficients of these obstructions all vanishes if we set

$$
\alpha_{1 / 2}=\frac{\kappa}{4} .
$$

Determination of $\omega$. In order to determine $\omega k_{a \Delta}$ we calculate all the terms that have the general structure " $A^{\star} F \zeta \zeta$ ". They only come from $\left(\hat{a}_{1}^{\text {int }}, \hat{a}_{1}^{\text {int }}\right),\left(a_{1}^{c c-\Lambda}, \tilde{a}_{1}^{c c}\right)$ and $\left(a_{2}^{\text {susy }}, \hat{a}_{1}^{G 1}\right)$. Focusing on the " $A^{\star} F \zeta \zeta$ " terms, we have

$$
\begin{aligned}
-\left.\frac{1}{2}\left(\omega \hat{a}_{1}^{\text {int }}, \omega \hat{a}_{1}^{\text {int }}\right)\right|_{A^{\star} F \zeta \zeta} & =-\frac{\omega^{2}}{2} A_{\mu}^{\star a} F_{\rho \sigma}^{b} \bar{\zeta}^{\Delta} \gamma^{\mu} \gamma^{\rho \sigma} \zeta^{\Omega} k_{a \Delta} k_{b \Omega}, \\
-\left.\frac{1}{2}\left(y a_{1}^{c c-\Lambda}, y \tilde{a}_{1}^{c c}\right)\right|_{A^{\star} F \zeta \zeta} & =\frac{\kappa^{2}}{32} A_{\mu}^{\star a} F_{\rho \sigma}^{b} \bar{\zeta}^{\Delta} \gamma^{\rho \sigma} \gamma^{\mu} \zeta^{\Omega} t_{a \Sigma \Delta} t_{b}{ }^{\Sigma} \Omega \\
-\left(\alpha_{3 / 2} a_{2}^{\text {susy }}, \alpha_{1} \hat{a}_{1}^{G 1}\right) & =-\frac{\kappa^{2}}{16} A_{a}^{\star \mu} F_{\mu \nu}^{a} \bar{\zeta}_{\Delta} \gamma^{\nu} \zeta^{\Delta} .
\end{aligned}
$$

By using the identities (A.5) and (A.6), the r.h.s. of the following equation

$$
\left.\frac{1}{\sqrt{-\bar{g}}}\left(\gamma b_{1}\right)\right|_{A^{\star} F \zeta \zeta}=-\left.\frac{1}{\sqrt{-\bar{g}}}\left(\delta b_{2}\right)\right|_{A^{\star} F \zeta \zeta}-\left.\frac{1}{2}\left(a_{1}, a_{1}\right)\right|_{A^{\star} F \zeta \zeta}-\left.\left(a_{2}, a_{1}\right)\right|_{A^{\star} F \zeta \zeta}+\left.\left(\nabla_{\mu} t_{1}^{\mu}\right)\right|_{A^{\star} F \zeta \zeta}
$$

is

$$
\begin{aligned}
& A_{a}^{\star \mu} F_{b \mu \nu} \bar{\zeta}_{(\Delta} \gamma^{\nu} \zeta_{\Omega)}\left(\frac{\kappa^{2}}{16} k^{a b} k^{\Delta \Omega}-\frac{\kappa^{2}}{16}\left(t^{a}\right)^{\Sigma(\Delta}\left(t^{|b|}\right)_{\Sigma}{ }^{\Omega)}-\omega^{2} k^{a(\Delta} k^{|b| \Omega)}\right) \\
& +A_{a}^{\star \mu} F_{b}^{\rho \sigma} \bar{\zeta}_{[\Delta} \gamma_{|\mu \rho \sigma|} \zeta_{\Omega]}\left(\frac{\kappa^{2}}{32}\left(t^{a}\right)^{\Sigma[\Delta}\left(t^{|b|}\right)_{\Sigma}{ }^{\Omega]}-\frac{\omega^{2}}{2} k^{a[\Delta} k^{|b| \Omega]}\right)+\nabla_{\mu} j_{1}^{\mu} .
\end{aligned}
$$

They give obstructions to finding $b_{1}$ because it is in $H(\gamma \mid d)$. Therefore the coefficient in front of these obstructions must vanish identically, providing us with the following constraints on the deformation structures:

$$
\begin{aligned}
\omega^{2} & =\frac{\kappa^{2}}{16}, \\
k^{a b} k^{\Delta \Omega} & =\left(t^{a}\right)^{\Sigma(\Delta}\left(t^{|b|}\right)_{\Sigma}{ }^{\Omega)}+k^{a(\Delta} k^{|b| \Omega)}, \\
k^{a[\Delta} k^{|b| \Omega]} & \left.=\left(t^{a}\right)^{\Sigma[\Delta}\left(t^{|b|}\right)_{\Sigma} \Omega\right]
\end{aligned}
$$

As regards the number of deformation parameters, after the resolution of the master equation at first order we had seven independent ones. The three constraints (3.70), (3.71) 
and (3.72) coming from the resolution of the master equation at second order and antifield number 2 (2.14) lower the number of deformation parameters down to four. Then the three constraints (3.81), (3.85) and (3.91) coming in the resolution at antifield number 1 (2.13) leave us with only one deformation parameter: $\kappa$. Of course, one can always take the $\lambda \longrightarrow 0$ limit of the final result and also view the cosmological constant as a deformation parameter. This is certainly the correct point of view when performing the deformation around flat spacetime, but less natural when performing the deformation around the $A d S_{4}$ background, where the cosmological constant can be arbitrarily large in absolute value.

Quadratic constraints. Let us summarise all the quadratic constraints on the normalised coefficients arising during the resolution of the master equation at second order:

$$
\begin{aligned}
k^{\Delta}{ }_{\Sigma} k^{\Sigma} & =k^{\Delta}, \\
f_{a[b}^{c} f^{a}{ }_{d e]} & =0, \\
2\left(t_{a}\right)^{\Delta}{ }_{\Sigma}\left(t_{b}\right)^{\Sigma} & =f^{c}{ }_{a b}\left(t_{c}\right)^{\Delta}, \\
\left(t^{a}\right)_{\Delta \Omega}\left(t_{a}\right)^{\Gamma \Sigma} & =2 k^{[\Gamma} k^{\Sigma]}{ }_{\Omega}, \\
k^{a}{ }_{c} k_{b}^{c} & =k^{a}{ }_{b}, \\
k^{a b} k^{\Delta \Omega} & =\left(t^{a}\right)^{\Sigma(\Delta}\left(t^{|b|}\right)_{\Sigma}{ }^{\Omega)}+k^{a(\Delta} k^{|b| \Omega)}, \\
k^{a[\Delta} k^{|b| \Omega]} & =\left(t^{a}\right)^{\Sigma[\Delta}\left(t^{|b|}\right)_{\Sigma}{ }^{\Omega]} .
\end{aligned}
$$

The constraints (3.94) are completely general in the sense that they are valid for arbitrary numbers of vectors and Rarita-Schwinger gauge fields, and independent of the matter content. The sole restriction is on the singleness of the graviton. On the other hand the constraints (3.95) will be modified by considering more than a single Majorana spin- $1 / 2$ field and some scalar fields.

Depending on the number of gravitini coupled to gravity, that is to say the rank of the matrix of coefficients $k^{\Delta}{ }_{\Sigma}$, different situations can arise as solution of the full set of constraints (3.94)-(3.95). In the following we detail the resolution of these constraints for a number of Rarita-Schwinger gauge fields coupled to gravity less than or equal to three: $\mathcal{N}_{4} \leqslant 3$. This will lead to the uniqueness of $\mathcal{N}_{4} \leqslant 3$ pure supergravities, when the field content is rich enough. The theory that describes the coupling of the $\mathcal{N}_{4}=1$ vector supermultiplet $(1,1 / 2)$ to the $\mathcal{N}_{4}=1$ supergravity multiplet $(2,3 / 2)$ is also included in the solution of the constraints when the menu of fields is adapted. A richer matter content is required to complete the specific constraints (3.95) in order to be able to include other matter-coupled supergravities and higher extended supergravity theories as solutions, a problem that we will treat elsewhere and that should reproduce, for instance, the models found in [31-33].

Uniqueness of $\mathcal{N}_{4}=3$ pure sugra. Firstly we will suppose that the matrix of coefficients $k^{\Delta} \Sigma_{\Sigma}$ has rank 3. Then from (3.73) we have $k^{\Delta}{ }_{\Sigma}=\delta_{\Sigma}^{\Delta}$ when $\Delta$ and $\Sigma$ run over $\{1,2,3\}$. The coefficients $k^{\Delta_{\Sigma}}$ are all null otherwise. It thus means that three of the massless spin- $\frac{3}{2}$ fields are coupled to gravity and therefore are gravitini. In order to study the uniqueness of $\mathcal{N}_{4}=3$ supergravity, let us see how three of the vector gauge fields couple 
to them. Concerning the Yang-Mills coupling of these three vector gauge fields, the two constraints (3.52) and (3.74) tell us that the coefficients $f_{a b}^{c}$ are the structure constants of a compact, real and semi-simple Lie group of dimension $n_{v}=3$, namely $\mathrm{SO}(3)$. Then the two constraints (3.75) and (3.76) tell us that the matrices $\left(t_{a}\right)^{\Delta \Omega}$ form a representation of dimension $\mathcal{N}_{4}=3$ of $\mathrm{SO}(3)$ and is a complete basis of the antisymmetric $\mathcal{N}_{4} \times \mathcal{N}_{4}=$ $3 \times 3$ matrices. All in all we have to take both $f_{a b}^{c}$ and $t_{a}{ }^{\Delta \Omega}$ to be the 3D Levi-Civita antisymmetric symbol and from now on we substitute the Latin indices $i, j, \ldots$ for the Latin indices $a, b, \ldots$ and for the upper-case Greek indices:

$$
\left(f_{a b}{ }^{c}, t_{a}^{\Delta \Omega}\right) \longrightarrow\left(\epsilon_{i j}{ }^{k}, \epsilon_{i}^{j k}\right) .
$$

With these notations, the remaining two constraints (3.92) and (3.93) that we have to solve can be written as

$$
\begin{aligned}
\delta^{i j} \delta^{k l} & =\epsilon_{p}^{i p\left(k \epsilon_{p}^{|j| l)}+k^{i(k} k^{|j| l)},\right.} \\
k^{i[k} k^{|j| l]} & =\epsilon_{p}^{i p[k} \epsilon_{p}^{|j| l]} .
\end{aligned}
$$

By use of the identity on the Levi-Civita symbols and Kronecker delta's

$$
\epsilon^{p_{1} \ldots p_{k} i_{1} \ldots i_{l}} \epsilon_{p_{1} \ldots p_{k} j_{1} \ldots j_{l}}=k ! l ! \delta_{j_{1}}^{\left[i_{1}\right.} \ldots \delta_{j_{l}}^{\left.i_{l}\right]}
$$

we can rewrite these two constraints in the following simple form

$$
k^{i(k} k^{|j| l)}=\delta^{i(k} \delta^{|j| l)}, \quad k^{i[k} k^{|j| l]}=\delta^{i[k} \delta^{|j| l]},
$$

where $k^{i j}$ is now the notation for the coefficients $k^{a \Delta}$. In this way, it is easy to see that all the solutions to (3.100) are given by $k^{i j}= \pm \delta^{i j}$. The \pm sign can be absorbed in the definition of $\omega$ in term of $\kappa$, cf. (3.91). Finally, we choose

$$
k^{i j}=\delta^{i j}
$$

which is exactly what appears in the cubic vertex involving the spins $\left(\frac{1}{2}, 1, \frac{3}{2}\right)$ in $\mathcal{N}_{4}=3$ pure supergravity $[17,20]$.

Uniqueness of $\mathcal{N}_{4}=2$ pure sugra. Since the $\mathcal{N}_{4}=2$ supergravity theory (without cosmological constant) is already discussed in the subsection 3.1, we will be very brief here. The solution of the constraints (3.94) and (3.95), which hold in the case where the cosmological constant is negative, can be analysed in the case where the matrix of coefficients $k^{\Delta}{ }_{\Sigma}$ is of rank 2, and has to be identified with $\delta_{\Sigma}^{\Delta}$ when the indices run over the two integer values $\{1,2\}$. The $\mathcal{N}_{4}=2$ supergravity theory [17] is recovered by considering the coupling with a single vector gauge field and no Majorana spin-1/2. Indeed the only non-trivial coefficients apart from $k^{\Delta} \Sigma_{\Sigma}$ are therefore $t_{a}{ }^{\Delta \Omega}$ and one can easily see that all the constraints are satisfied if and only if it is identified with $\epsilon^{\Delta \Omega}$. 
$\mathcal{N}_{4}=1$ sugras. Finally we will study the case where the matrix of coefficients $k^{\Delta_{\Sigma}}$ has rank 1. The solution of the constraint (3.73) therefore is $k_{1}^{1}=1$ as sole non-zero component of $k^{\Delta} \Sigma_{\Sigma}$. The simplest solution to all the constraints is to take all the other coefficients to vanish. This almost trivial solution is possible only when a single real Rarita-Schwinger gauge field couples to gravity because the r.h.s. of (3.76) vanishes only in this case. This yields the $\mathcal{N}_{4}=1$ supergravity theory, with [22] and without [34, 35] cosmological constant.

Still in the case where there is a single gravitino, another solution can be found. From (3.76) we will still set the coefficients $t_{a}{ }^{\Delta \Omega}$ to zero. Then the constraints (3.95) become

$$
k_{c}^{a} k_{b}^{c}=k_{b}^{a}, \quad k^{a b} k^{\Delta \Omega}=k^{a(\Delta} k^{|b| \Omega)}, \quad k^{a[\Delta} k^{|b| \Omega]}=0,
$$

that we solve by considering that a single vector gauge field couples to gravity by taking $k_{b}^{a}=1$ for $a=b=1$, and $k_{b}^{a}=0$ otherwise. Subsequently, the coefficients $k^{a \Delta}$ have to be 1 when $a=1$ and $\Delta=1$, and 0 otherwise. This leads to a theory, with negative cosmological constant terms, of a vector supermultiplet $(1,1 / 2)$ coupled to the supergravity multiplet $(2,3 / 2)$ through the vertex (3.60). The flat limit of this model is none other than the model of $[16]$.

Summary and higher-order deformations. To summarise, we reproduced all the cubic vertices of, respectively, $\mathcal{N}_{4}=3, \mathcal{N}_{4}=2$ and $\mathcal{N}_{4}=1$ pure supergravities, as well as the cubic vertices of the model of [16] including extensions by cosmological constant terms that had not been discussed before in the literature, to the best of our knowledge. This was done by setting $W=W_{0}+g W_{1}(\tilde{c})+\mathcal{O}\left(g^{2}\right)$ for a set of infinitesimal parameters collectively denoted by $g$ and for some coupling constants collectively denoted by $\tilde{c}, W_{0}$ being the solution to the master equation $\left(W_{0}, W_{0}\right)=0$ for the free theory, and by classifying the general solution to the equation $(W, W)=0$ at first order in $g$, under the assumptions stated in our theorem. We denote the general solution by $W_{1}^{\text {sugra }}(\tilde{c})$ as it contains, in its antifield-independent piece, all the cubic vertices of the supergravity theories with $\mathcal{N}_{4} \leqslant 3$ listed above. It depends linearly on the parameters $\tilde{c}$ that must obey linear constraints, as for example $f_{a b c}=f_{[a b c]}$ for the Yang-Mills deformation.

Having classified the solution space for $W_{1}$, we then turned to the master equation at second order in $g,\left(W_{0}, W_{2}\right)+\frac{1}{2}\left(W_{1}, W_{1}\right)=0$, and solved it for $W_{2}$ at antifield numbers 2 and 1. This enabled us to find quadratic constraints on the parameters $\tilde{c}$, allowing four classes of nontrivial solutions, depending on the rank of the matrices $k^{\Delta}{ }_{\Gamma}$ and $k_{b}^{a}$ that are part of the constants denoted by $\tilde{c}$. The solution $W_{2}$ at antifield number zero will contain the usual quartic vertices of the various supergravity theories discussed above, although we have not computed them explicitly, as this is not necessary to prove the uniqueness, under the assumptions of our theorem, of the aforementioned supergravity theories with $\mathcal{N}_{4} \leqslant 3$.

Indeed, knowing $W_{1}^{\text {sugra }}(\tilde{c})$, the general solution of the order- $g$ equation $\left(W_{0}, W_{1}\right)=0$ - under the assumptions stated in our theorem - and knowing the full theories that precisely reproduce these first-order interactions, one can deduce that the general solution of the master equation, to all orders, will just produce the nonlinear theories whose cubic vertices are parts of our general solution $W_{1}$ at antifield number zero. We can repeat, in our current context, the general argument of the section 7 of [11] leading to the uniqueness of 
Einstein-Hilbert's action from the uniqueness of its cubic vertex. From the results obtained so far, we have that $W=W_{0}+g W_{1}^{\text {sugra }}(\tilde{c})+g^{2} W_{2}+\ldots$, where the various parameters, collectively denoted by $\tilde{c}$, solve the quadratic constraints (3.94) and (3.95). Therefore, depending on the class of solution of these quadratic constraints, $W_{1}^{\text {sugra }}(\tilde{c})$ denotes the solution of the master equation at first order in interactions, for one of the four $\mathcal{N}_{4} \leqslant 3$ supergravity theories recalled above.

The functional $W_{2}$ has to solve $s W_{2}=-\frac{1}{2}\left(W_{1}^{\text {sugra }}(\tilde{c}), W_{1}^{\text {sugra }}(\tilde{c})\right)$, therefore one can $\operatorname{set}^{7}$ $W_{2}=W_{2}^{\text {sugra }}\left(\tilde{c}^{2}\right)+W_{2}^{\prime}$, for $W_{2}^{\text {sugra }}$ the second-order solution of the classical master equation for the corresponding nonlinear $\mathcal{N}_{4} \leqslant 3$ supergravity theory that we know exists. The functional $W_{2}^{\prime}$ must solve the homogeneous equation $\left(W_{0}, W_{2}^{\prime}\right)=0$, whose general solution (under the assumptions stated in our theorem) is $W_{2}^{\prime}=W_{1}^{\text {sugra }}\left(c^{\prime}\right)$ featuring some parameters $c^{\prime}$ that solve the same linear constraints as the parameters $\tilde{c}$. The equation for $W_{3}$ is then $\left(W_{0}, W_{3}\right)=-\left(W_{2}, W_{1}^{\text {sugra }}(\tilde{c})\right)=-\left(W_{2}^{\text {sugra }}\left(\tilde{c}^{2}\right), W_{1}^{\text {sugra }}(\tilde{c})\right)-\left(W_{1}^{\text {sugra }}(\tilde{c}), W_{1}^{\text {sugra }}\left(c^{\prime}\right)\right)$. We can set $W_{3}=W_{3}^{\text {sugra }}\left(\tilde{c}^{3}\right)+W_{3}^{\prime}$ where $W_{3}^{\prime}$ must solve

$$
\left(W_{0}, W_{3}^{\prime}\right)=-\left(W_{1}^{\text {sugra }}(\tilde{c}), W_{1}^{\text {sugra }}\left(c^{\prime}\right)\right) .
$$

The right-hand side of the latter equation can be $s$-exact only if the parameters $\bar{c}:=\tilde{c}+g c^{\prime}$ satisfy the quadratic constraints (3.94) and (3.95), to the relevant order in $g$. From $W_{2}^{\prime}=$ $W_{1}^{\text {sugra }}\left(c^{\prime}\right)$, eq. (3.103) and eq. (3.62), one finds that $W_{3}^{\prime}=2 W_{2}^{\text {sugra }}\left(\tilde{c} c^{\prime}\right)$ up to a solution $W_{3}{ }^{\prime \prime}$ of the equation $\left(W_{0}, W_{3}{ }^{\prime \prime}\right)=0$, i.e. $W_{3}{ }^{\prime \prime}=W_{1}^{\text {sugra }}\left(c^{\prime \prime}\right)$. Putting things together and continuing to higher orders, one obtains $W^{\text {sugra }}=W_{0}+W_{1}^{\text {sugra }}(c)+W_{2}^{\text {sugra }}\left(c^{2}\right)+\ldots$ that solves $\left(W^{\text {sugra }}, W^{\text {sugra }}\right)=0$ and depends on the coupling constants $c=g \tilde{c}+g^{2} c^{\prime}+g^{3} c^{\prime \prime}+\ldots$. This proves the uniqueness of the corresponding four supergravity theories discussed above.

Therefore, knowing the existence of the complete supergravity theories whose cubic vertices we classified and identified above is enough to deduce the uniqueness of the corresponding nonlinear theories. Still, it is interesting to exhibit the quartic vertices from the cohomological approach. We have already provided quartic terms in the structure of the gauge algebra, including the typical on-shell closure terms that are a landmark of all supergravity theories without auxiliary fields. We intend to give them all, as well as the typical quartic vertices, in a forthcoming publication, although we stress that this is not at all necessary for the proof of the uniqueness of these models.

\section{Conclusions and perspectives}

In this note we used the purely algebraic reformulation [7] of the problem of introducing consistent interactions among (gauge) fields in order to study theories that couple a set of massless spin- $\frac{3}{2}$ fields to a set of Maxwell fields in four dimensions. This work can therefore be seen as the extension of the analyses performed in [11] and [21]. We recovered and proved the uniqueness of all the known, pure supergravity theories in four dimensions with $\mathcal{N}_{4} \leqslant 3$ and also obtained strong necessary constraints on the gaugings of higher mattercoupled or more extended supergravity models. In a future work, we will increase the menu

\footnotetext{
${ }^{7}$ The notation $W_{2}^{\text {sugra }}\left(\tilde{c}^{2}\right)$ is meant to indicate that $W_{2}^{\text {sugra }}$ depends quadratically on the parameters.
} 
of matter fields and study the solution of these constraints. In perturbation around $A d S_{4}$ background for pure $\mathcal{N}_{4} \geqslant 4$ models, it will be interesting to see how both the possible gaugings and exponentials of the scalar fields appear in the appropriate cohomologies. An interesting perspective for future work would also be to increase the spacetime dimension and consider $p$-form gauge fields in the spectrum, so as to understand, from a BRSTcohomological point of view, the role of the latter fields in higher-dimensional, extended gauged supergravities.

\section{Acknowledgments}

We thank Glenn Barnich, Marc Henneaux, Victor Lekeu and Arash Ranjbar for discussions. Nicolas Boulanger thanks the Laboratoire de Physique Théorique de l'École Normale Supérieure de Paris for kind hospitality, while Bernard Julia thanks the Groupe de Mécanique et Gravitation of UMONS for hospitality. This work was partially supported by FNRS-Belgium (convention PDR T.1025.14).

\section{A Conventions and some Diracology}

In this appendix, we spell out our conventions for spinors in Minkowski space $\mathbb{R}^{1,3}$ and recall some known identities on Dirac matrices. A useful compendium of tools for supersymmetry, including conventions and identities on Dirac matrices in various dimensions, can be found in $[36]$.

Clifford Algebra. Our convention for the Levi-Civita tensor $\epsilon^{a b c d}$ in Cartesian coordinates is such that $\epsilon^{0123}=1=-\epsilon_{0123}$. We use the mostly plus convention for the Minkowski metric in Cartesian coordinates: $\eta=\operatorname{diag}(-,+,+,+)$. The Clifford algebra

$$
\left\{\gamma^{a}, \gamma^{b}\right\}=2 \eta^{a b} \mathbb{1}_{4}
$$

reads $\left\{\gamma^{a}, \gamma^{b}\right\}_{A}^{B}=2 \eta^{a b} \delta_{A}^{B}$ in components, with $A, B=1, \ldots, 4$ and where we take as a convention that the components of the gamma matrix $\gamma^{a}$ are $\left(\gamma^{a}\right)_{A}{ }^{B}$.

Dirac adjoint. Our conventions for the complex, transpose and Hermitian conjugates of a matrix $M$ in terms of its components $M_{A}{ }^{B}$ are $\left[M^{*}\right]^{A^{\prime}}{ }_{B^{\prime}}=\left(M_{A}{ }^{B}\right)^{*},\left[M^{T}\right]^{B}{ }_{A}=M_{A}{ }^{B}$ and $\left[M^{\dagger}\right]_{B^{\prime}} A^{\prime}=\left(M_{A}^{B}\right)^{*}$. The components of a Dirac spinor are denoted by $\psi_{A}, A=1, \ldots, 4$. A sesquilinear form on the space of Dirac spinor is introduced, $\beta_{A^{\prime}}{ }^{B}:=i\left(\gamma^{0}\right)_{A}{ }^{B}$, from which the Dirac adjoint of the Dirac spinor $\psi_{A}$ is defined, as usual, by $\bar{\psi}^{A}:=\left(\psi^{\dagger}\right)^{B^{\prime}} \beta_{B^{\prime}}{ }^{A}$ in components, or $\bar{\psi}=\psi^{\dagger} \beta$ in matrix notation.

Different sets of matrices obeying the Clifford algebra (A.1) are related by a change of basis. As the matrices $-\left(\gamma^{a}\right)^{\dagger}$ also satisfy the relation (A.1), they can be obtained from a change of basis starting from $\gamma^{a}$. Indeed, one has $\beta \gamma^{a} \beta^{-1}=-\left(\gamma^{a}\right)^{\dagger}$. The matrix $\gamma^{5}$ is defined as $\gamma^{5}:=i \gamma^{0} \gamma^{1} \gamma^{2} \gamma^{3}$. It obeys $\left(\gamma^{5}\right)^{2}=\mathbb{1}_{4}$ and anticommutes with the four matrices of the Clifford algebra $\gamma^{a}$. One also defines $\gamma^{a_{1} a_{2} \ldots a_{n}}:=\gamma^{\left[a_{1}\right.} \gamma^{a_{2}} \ldots \gamma^{\left.a_{n}\right]}$, where

$$
\left[a_{1} \ldots a_{n}\right]=\frac{1}{n !} \sum_{\sigma \in S_{n}}(-1)^{\operatorname{Sgn}(\sigma)} a_{\sigma(1)} \ldots a_{\sigma(n)}
$$


defines the complete antisymmmetrization with strength one. We denote the matrices $\gamma^{a_{1} a_{2} \ldots a_{n}}$ by $\gamma^{a[n]}$, for the sake of brevity. As usual, one gathers together these 16 matrices in the following set $\left\{M_{I}\right\}_{I=1 \ldots 16}=\left\{\mathbb{1}_{4}, \gamma^{a}, \gamma^{a[2]}, \gamma^{a[3]}, \gamma^{5}\right\}$ that forms a basis of the $4 \times 4$ complex matrices.

Majorana conjugate. Another representation of the Clifford algebra is given by minus the transposed Dirac matrices $-\left(\gamma^{a}\right)^{T}$ with components $-\left[\left(\gamma^{a}\right)^{T}\right]_{B}^{A}$. The charge conjugation matrix $C^{A B}$ realizes the similitude

$$
C \gamma^{a} C^{-1}=-\left(\gamma^{a}\right)^{T}
$$

In even dimension it is possible to choose $C$ to be unitary $C^{\dagger}=C^{-1}$ and antisymmetric $C^{T}=-C$.

Starting from the 16 basis matrices $M_{I}$ defined above, one deduces from (A.2) and the antisymmetry of the charge conjugation matrix that $C \gamma^{a}$ and $C \gamma^{a[2]}$ are symmetric matrices, whereas $C \gamma^{a[3]}, C \gamma^{5}$ and $C$ are antisymmetric. The charge conjugation matrix $C$ is instrumental in providing a definition of the Majorana conjugate $\widetilde{\psi}:=\psi^{T} C$, or $\widetilde{\psi}^{A}=$ $\psi_{B} C^{B A}=-C^{A B} \psi_{B}$ in components. A Majorana spinor is a spinor such that its Dirac conjugate $\bar{\psi}^{A}$ is equal to its Majorana conjugate $\widetilde{\psi}^{A}$.

Identities relative to the Dirac matrices. We collect here various identities that we needed in our calculations:

$$
\begin{aligned}
\gamma^{m n r} \gamma^{a b} & \equiv\left(\gamma^{m n r} \gamma^{a b}\right)+\left(\gamma^{m n r} \gamma^{a b}\right), \text { where } \\
\left(\gamma^{(1)} \gamma^{a b}\right) & :=-6 \eta^{a[m} \eta^{|b| n} \gamma^{r]} \text { and }\left(\gamma^{m n r} \gamma^{a b}\right):=3\left(\eta^{a[m} \gamma^{n r] b}-\eta^{b[m} \gamma^{n r] a}\right) .
\end{aligned}
$$

Moreover,

$$
\begin{aligned}
\gamma_{m n p} \gamma^{p} & =2 \gamma_{m n} \\
\gamma_{n p} \gamma_{m} & =\gamma_{n p m}-2 \eta_{m[n} \gamma_{p]}, \\
\gamma_{n} \gamma_{a b} & =\gamma_{n a b}+2 \eta_{n[a} \gamma_{b]} \\
{\left[\gamma_{n}, \gamma_{a b}\right] } & =4 \eta_{n[a} \gamma_{b]} \\
\gamma_{a b} \gamma_{m n} & =-i \gamma^{5} \epsilon_{a b m n}-2\left(\eta_{m[a} \gamma_{b] n}-\eta_{n[a} \gamma_{b] m}\right)-2 \eta_{m[a} \eta_{b] n} \mathbb{1} .
\end{aligned}
$$

Finally,

$$
\gamma_{m n p}=i \epsilon_{m n p q} \gamma^{q} \gamma^{5}=-i \epsilon_{m n p q} \gamma^{5} \gamma^{q}
$$

\section{B Appearance of conserved currents in gaugings}

In this appendix we make some comments on the relation between what is known as the Noether procedure for introducing couplings among fields and the BRST-BV reformulation $[7,37$ ] of the consistent coupling procedure explained e.g. in [10]. More precisely, in 
the present paper we have followed the techniques and used some general theorems proved in $[23,24]$. According to this procedure and the equations (2.9)-(2.11) for the determination of the first-order interactions, one can classify all such infinitesimal interactions, represented in the Lagrangian by $a_{0}$, according to whether (i) $a_{2}=0=a_{1}$, (ii) $a_{2}=0$ but $a_{1} \neq 0$ and (iii) $a_{2} \neq 0$. In all three cases, one can write the vertex $a_{0}$ in the Noether form "gauge field times conserved current".

From the results we obtained in the body of the paper, we now discuss these 3 cases in a way that closely follows the exposition made in section 3.2 of [13], but this time for a spectrum of fields that also contains spin-2, spin- $\frac{3}{2}$ and spin- $\frac{1}{2}$ fields. A detailed discussion of the pure spin-2 case was given in section 11 of [11].

(i) $\boldsymbol{a}_{2}=\mathbf{0}=\boldsymbol{a}_{1}$. In these cases the vertices and hence the currents can be written as functions of the (derivatives of the) field strengths of the various fields and of the spin- $\frac{1}{2}$ field. Moreover, the currents are strictly conserved, in the sense that they do not require the field equations of the undeformed Lagrangian for the divergence to vanish. In our analysis where we imposed the upper limit of two derivatives in the final Lagrangian, such vertices play no role;

(ii) $a_{2}=0, a_{1} \neq 0$. In these cases, the classification of vertices coming from all the possible $\hat{a}_{1}$ such that $\gamma \hat{a}_{1}=0$ has produced interactions of the Noether type where the currents are gauge invariant and conserved by virtue of the field equations of the free theory. With our bound on the maximal number of derivatives in the Lagrangian, we listed many candidates $\hat{a}_{1}$ but only a very few of them can give rise to vertices. They were respectively denoted by $\hat{a}_{1}^{\text {int }}, \hat{a}_{1}^{G 1}$ and $\hat{a}_{1}^{G 1 / 2}$. The corresponding currents are, respectively, equivalent (up to trivial terms and on-shell vanishing expressions) to $J_{\text {int. }{ }_{\Delta}}^{\mu}=\frac{1}{2} k_{a \Delta} F_{\alpha \beta}^{a} \gamma^{\alpha \beta} \gamma^{\mu} \chi, J_{G 1}^{\mu \nu}=\frac{1}{2} k^{a b}\left(F_{a}^{\mu \rho} F_{b \rho}^{\nu}-\frac{1}{4} \eta^{\mu \nu} F_{a}^{\alpha \beta} F_{b \alpha \beta}\right)$, and $J_{G 1 / 2}^{\mu \nu}=\partial_{\mu} \bar{\chi} \gamma^{(\mu} \partial^{\nu)} \chi$. They correspond to rigid symmetries of the free theory where the fields appear through gauge-invariant expressions, as can be read off from the expressions for $\hat{a}_{1}^{\text {int }}, \hat{a}_{1}^{G 1}$ and $\hat{a}_{1}^{G 1 / 2}$. With hindsight, the corresponding interactions result from the gauging of the corresponding rigid symmetries as we recall below;

(iii) $\boldsymbol{a}_{\mathbf{2}} \neq \mathbf{0}$. This third case is probably the most interesting one, as it induces non-abelian deformations of the gauge structure of the initial abelian theory, leading to a Noether coupling where not only the current is non-invariant, but also the divergence of the current. An example from our analysis is the current associated with the algebradeforming term $a_{2}^{(4)}=k_{\Delta \Omega}^{(4)} \mathcal{C}^{\star} \bar{\zeta}^{[\Delta} \zeta^{\Omega]}$, giving rise to the deformation of the gauge transformations encoded in $a_{1}^{(4)}=-4 t_{\Delta \Omega}^{(4)} A^{\star \mu} \bar{\psi}_{\mu}^{\Delta} \zeta^{\Omega}+t_{\Delta \Omega} \psi_{\sigma}^{\star \Delta} F_{\mu \nu} \gamma^{\mu \nu} \gamma^{\sigma} \zeta^{\Omega}$ and the conserved current $J_{\Delta}^{\mu}=\epsilon_{\Delta \Omega}\left(F^{\mu \nu} \mathbb{1}-i \gamma^{5}(* F)^{\mu \nu}\right) \psi_{\nu}^{\Omega}$. It is precisely because the corresponding $a_{1}$ is not gauge invariant that an $a_{2}$ term is needed, responsible for the deformation of the gauge structure. These vertices are related $[23,24,38]$ to the existence, in the initial free theory, of on-shell $d$-closed $n-2$-forms, that are $* F^{a}$, $a=1, \ldots, n_{v}$ in the spin- 1 case, equivalently conserved 2 -forms $F^{a}$. In the spin- $\frac{3}{2}$ case, the components of the conserved two-forms are $\bar{\psi}_{\rho}^{\Delta} \gamma^{\mu \nu \rho}$, for $\Delta \in\left\{1, \ldots, \mathcal{N}_{4}\right\}$. In the 
spin-2 case, the set of forms corresponds to a vector-valued two-form with components $\Phi_{\left.\mu \nu\right|^{\alpha}}=-\left.\Phi_{\nu \mu}\right|^{\alpha}$ given in eq. (4.11) of [11]. Notice the difference between spin-1 and spin-2: in the former case, the $n-2$ forms $* F^{a}$ are gauge invariant, whereas it is not true in the latter case since $\Phi_{\mu \nu \mid}{ }^{\alpha}$ is built out of first derivatives of the Fierz-Pauli field.

Actually in [38] it was found that an abelian local invariance leads to an on-shell conserved 2-form "current" whenever the gauge parameter is a closed 1-form ie when the usual gauge transformation parameter does not appear undifferentiated (the latter restriction is generalized by the so-called Killing condition below). This leads as in the global symmetry case to a general formula for the Noether-like conserved 2-form (e.g. $F^{a}$ ). We should take note that after a first-order abelian or non abelian deformation the equation of motion of the vector gauge field implies that the differential of the abelian $* F^{a}$ is proportional to an ordinary (on-shell closed) 3-form that corresponds to a global invariance by the inverse Noether theorem.

Supposing we know (a) an infinitesimal deformation of the solution of the master equation that deforms the gauge transformation laws and (b) the existence of a Killing parameter of the initial theory, then one can identify a rigid symmetry of the initial theory. Indeed, let the initial theory $S_{0}\left[\varphi^{i}\right]=\int d^{4} x \mathcal{L}_{0}$ be invariant under $\delta_{0} \varphi^{i}=R^{(0) i}{ }_{\alpha} \varepsilon^{\alpha}$. The existence of $W_{1}=\int d^{4} x\left(\mathcal{L}_{1}+a_{1}+a_{2}\right)$ - where $a_{2}$ can possibly vanish - implies that $\delta_{0} S_{1}+\delta_{1} S_{0}=0$, which is nothing but a rewriting of the integral of (2.9). There, one has $\delta_{1} \varphi^{i}=R^{(1) i}{ }_{\alpha} \varepsilon^{\alpha}$ and $S_{1}=\int d^{4} x \mathcal{L}_{1}$. Killing parameters $\bar{\varepsilon}^{\alpha}$ of the initial theory obey $\bar{\delta}_{0} \varphi^{i}:=R^{(0) i}{ }_{\alpha} \bar{\varepsilon}^{\alpha}=0$. Therefore, computing $\bar{\delta}_{0} S_{1}+\bar{\delta}_{1} S_{0}$ yields $0=\int d^{4} x \bar{\delta}_{1} \varphi^{i} \frac{\delta \mathcal{L}_{0}}{\delta \varphi^{i}}$, from which follows the rigid symmetry of the initial theory $S_{0}$ under $\bar{\delta}_{1} \varphi^{i}=R^{(1) i}{ }_{\alpha} \bar{\varepsilon}^{\alpha}$. Therefore, the gauging of this rigid symmetry can retrospectively be viewed as giving the infinitesimal deformation encoded in $W_{1}$.

In the case the fields $\varphi^{i}$ are the vector fields $A_{\mu}^{a}$, gauging the above rigid symmetry gives $\delta_{1} S_{0}=-\int d^{4} x J_{a}^{\mu} \partial_{\mu} \varepsilon^{a}$ featuring the Noether current $J_{a}^{\mu}$ associated with the rigid symmetry $\bar{\delta}_{1} A_{\mu}^{a}=R^{(1) a}{ }_{\mu b} \bar{\varepsilon}^{b}$ where $\bar{\varepsilon}^{b}$ are $n_{v}$ constants. On the other hand, we know that there exists $S_{1}$ such that $\delta_{1} S_{0}+\delta_{0} S_{1}=0$. From the knowledge of $R^{(0) i}{ }_{\alpha}$ for abelian vector fields, one easily deduces that $J_{\mu}^{a}+\partial^{\nu} S_{\mu \nu}^{a}=\frac{\delta \mathcal{L}_{1}}{\delta A_{a}^{\mu}}$ where $S_{\mu \nu}^{a}=-S_{\nu \mu}^{a}$. Therefore, the deformation of the action $S_{0}$ can be written $S_{1}=\int d^{4} x \mathcal{L}_{1}=\int d^{4} x \int_{0}^{1} d t A_{a}^{\mu} \frac{\delta \mathcal{L}_{1}}{\delta A_{a}^{\mu}}(t A)$ from which $S_{1}$ appears to be proportional to the expression $\int d^{4} x A_{\mu}^{a}\left(J_{a}^{\mu}+\partial_{\nu} S_{a}^{\mu \nu}\right)$. In case $\mathcal{L}_{1}$ is cubic, one finds $S_{1}=\frac{1}{3} \int d^{4} x A_{a}^{\mu} J_{\mu}^{a}$, showing the analogy with a Noether coupling, but also showing the difference in the overall coefficient.

In more detail, for a set of Maxwell gauge fields, the rigid rotational symmetry $\bar{\delta}_{1} A_{\mu}^{a}=$ $f_{\mid b c}^{a} A_{\mu}^{b} \bar{\epsilon}^{c}$ with $f_{a \mid b c}=-f_{b \mid a c}$ leads to the canonical Noether current $J_{c}^{\mu}=F_{a}^{\mu \nu} f_{b c}^{a} A_{\nu}^{b}$. On the other hand, the Yang-Mills cubic vertex reads $\mathcal{L}_{1}=-\frac{1}{2} F^{\mu \nu} f^{a}{ }_{b c} A_{\mu}^{b} A_{\nu}^{c}$, with totally antisymmetric constants $f_{a \mid b c}$, from which one derives $\frac{\delta \mathcal{L}_{1}}{\delta A_{\mu}^{a}}=J_{a}^{\mu}+\partial_{\nu} S_{a}^{\mu \nu}$ where $S_{\mu \nu}^{a}=$ $-S_{\nu \mu}^{a}=-f_{b c}^{a} A_{\mu}^{b} A_{\nu}^{c}$. The cubic action is therefore given by $S_{1}\left[A_{\mu}^{a}\right]=\frac{1}{3} \int d^{4} x A_{\mu}^{a} \frac{\delta \mathcal{L}_{1}}{\delta A_{\mu}^{a}}=$ $\frac{1}{2} \int d^{4} x A_{\mu}^{a} J_{a}^{\mu}$, where one notices the non-canonical factor $1 / 2$ due to the fact that the so-called "trivial" current $\partial_{\nu} S_{a}^{\mu \nu}$ does contribute to $S_{1}$.

The analysis proceeds similarly for the cases where $\varphi^{i}$ are massless spin- $\frac{3}{2}$ or massless spin-2 fields. One deduces from $\mathcal{L}_{1}$ the existence of rigid symmetries with on-shell conserved 
currents $J_{I}^{\mu}$ where $I=\alpha$ in the massless spin- 2 case and $I=(A, \Delta)$ in the massless spin- $\frac{3}{2}$ case, such that $J^{\mu}{ }_{\alpha} \propto \frac{\delta \mathcal{L}_{1}}{\delta h_{\mu}{ }^{\alpha}}$ and $J^{\mu}{ }_{A \Delta} \propto \frac{\delta \mathcal{L}_{1}}{\delta \bar{\psi}_{\mu} A \Delta}$, up to the addition of "trivial" currents and on-shell vanishing terms.

Finally, let us stress that the presence of conserved 2 forms is tied to the presence of free abelian gauge fields, be them of spin 1 or 2. Moreover, in the absence of free abelian gauge fields, the conserved currents of Yang-Mills gauge models coupled to matter fields can always be redefined so as to be gauge invariant as shown in [39].

Open Access. This article is distributed under the terms of the Creative Commons Attribution License (CC-BY 4.0), which permits any use, distribution and reproduction in any medium, provided the original author(s) and source are credited.

\section{References}

[1] G. Velo and D. Zwanziger, Propagation and quantization of Rarita-Schwinger waves in an external electromagnetic potential, Phys. Rev. 186 (1969) 1337 [INSPIRE].

[2] K. Johnson and E.C.G. Sudarshan, Inconsistency of the local field theory of charged spin 3/2 particles, Annals Phys. 13 (1961) 126 [INSPIRE].

[3] S.L. Adler, Classical Gauged Massless Rarita-Schwinger Fields, Phys. Rev. D 92 (2015) 085022 [arXiv: 1508.03380] [INSPIRE].

[4] S.L. Adler, Quantized Gauged Massless Rarita-Schwinger Fields, Phys. Rev. D 92 (2015) 085023 [arXiv: 1508.03382] [INSPIRE].

[5] W. Rarita and J. Schwinger, On a theory of particles with half integral spin, Phys. Rev. 60 (1941) 61 [INSPIRE].

[6] S.L. Adler, M. Henneaux and P. Pais, Canonical Field Anticommutators in the Extended Gauged Rarita-Schwinger Theory, Phys. Rev. D 96 (2017) 085005 [arXiv:1708.03588] [INSPIRE].

[7] G. Barnich and M. Henneaux, Consistent couplings between fields with a gauge freedom and deformations of the master equation, Phys. Lett. B 311 (1993) 123 [hep-th/9304057] [INSPIRE].

[8] I.A. Batalin and G.A. Vilkovisky, Gauge Algebra and Quantization, Phys. Lett. B 102 (1981) 27 [INSPIRE].

[9] I.A. Batalin and G.A. Vilkovisky, Quantization of Gauge Theories with Linearly Dependent Generators, Phys. Rev. D 28 (1983) 2567 [Erratum ibid. D 30 (1984) 508] [INSPIRE].

[10] F.A. Berends, G.J.H. Burgers and H. van Dam, On the Theoretical Problems in Constructing Interactions Involving Higher Spin Massless Particles, Nucl. Phys. B 260 (1985) 295 [INSPIRE].

[11] N. Boulanger, T. Damour, L. Gualtieri and M. Henneaux, Inconsistency of interacting, multigraviton theories, Nucl. Phys. B 597 (2001) 127 [hep-th/0007220] [INSPIRE].

[12] M. Henneaux, G. Lucena Gómez and R. Rahman, Higher-Spin Fermionic Gauge Fields and Their Electromagnetic Coupling, JHEP 08 (2012) 093 [arXiv: 1206.1048] [INSPIRE].

[13] G. Barnich, N. Boulanger, M. Henneaux, B. Julia, V. Lekeu and A. Ranjbar, Deformations of vector-scalar models, JHEP 02 (2018) 064 [arXiv: 1712.08126] [INSPIRE]. 
[14] C. Bizdadea and S.-O. Saliu, Gauge-invariant massive BF models, Eur. Phys. J. C 76 (2016) 65 [arXiv: 1511.04684] [INSPIRE].

[15] M. Henneaux, G. Lucena Gómez and R. Rahman, Gravitational Interactions of Higher-Spin Fermions, JHEP 01 (2014) 087 [arXiv:1310.5152] [InSPIRE].

[16] S. Ferrara, J. Scherk and P. van Nieuwenhuizen, Locally Supersymmetric Maxwell-Einstein Theory, Phys. Rev. Lett. 37 (1976) 1035 [InSPIRE].

[17] D.Z. Freedman and A.K. Das, Gauge Internal Symmetry in Extended Supergravity, Nucl. Phys. B 120 (1977) 221 [INSPIRE].

[18] E.S. Fradkin and M.A. Vasiliev, SO(2) supergravity with minimal electromagnetic interaction, 1.5-order formalism and the explicit form of the structure coefficients, Lebedev Institute Preprint 197 (1976).

[19] S. Ferrara and P. van Nieuwenhuizen, Consistent Supergravity with Complex Spin 3/2 Gauge Fields, Phys. Rev. Lett. 37 (1976) 1669 [inSPIRE].

[20] D.Z. Freedman, SO(3) Invariant Extended Supergravity, Phys. Rev. Lett. 38 (1977) 105 [INSPIRE].

[21] N. Boulanger and M. Esole, A note on the uniqueness of $D=4, N=1$ supergravity, Class. Quant. Grav. 19 (2002) 2107 [gr-qc/0110072] [INSPIRE].

[22] P.K. Townsend, Cosmological Constant in Supergravity, Phys. Rev. D 15 (1977) 2802 [INSPIRE].

[23] G. Barnich, F. Brandt and M. Henneaux, Local BRST cohomology in the antifield formalism. 1. General theorems, Commun. Math. Phys. 174 (1995) 57 [hep-th/9405109] [InSPIRE].

[24] G. Barnich, F. Brandt and M. Henneaux, Local BRST cohomology in the antifield formalism. II. Application to Yang-Mills theory, Commun. Math. Phys. 174 (1995) 93 [hep-th/9405194] [INSPIRE].

[25] M. Henneaux and C. Teitelboim, Quantization of gauge systems, Princeton University Press, Princeton, U.S.A., (1992).

[26] J. Gomis, J. Paris and S. Samuel, Antibracket, antifields and gauge theory quantization, Phys. Rept. 259 (1995) 1 [hep-th/9412228] [INSPIRE].

[27] L. Traina, Théorie de supergravité étendue $n=2$ en dimension 4, MSc Thesis, Université de Mons - UMONS, (2016), http://hosting.umons.ac.be/php/mecagrav/english/traina.php.

[28] K. Pilch, P. van Nieuwenhuizen and M.F. Sohnius, de Sitter Superalgebras and Supergravity, Commun. Math. Phys. 98 (1985) 105 [INSPIRE].

[29] E.A. Bergshoeff, D.Z. Freedman, R. Kallosh and A. Van Proeyen, Pure de Sitter Supergravity, Phys. Rev. D 92 (2015) 085040 [Erratum ibid. D 93 (2016) 069901] [arXiv: 1507.08264] [INSPIRE].

[30] G. Barnich, M. Henneaux and R. Tatar, Consistent interactions between gauge fields and the local BRST cohomology: The example of Yang-Mills models, Int. J. Mod. Phys. D 3 (1994) 139 [hep-th/9307155] [INSPIRE].

[31] E. Cremmer, B. Julia, J. Scherk, S. Ferrara, L. Girardello and P. van Nieuwenhuizen, Spontaneous Symmetry Breaking and Higgs Effect in Supergravity Without Cosmological Constant, Nucl. Phys. B 147 (1979) 105 [inSPIRE]. 
[32] E. Cremmer, S. Ferrara, L. Girardello and A. Van Proeyen, Yang-Mills Theories with Local Supersymmetry: Lagrangian, Transformation Laws and SuperHiggs Effect, Nucl. Phys. B 212 (1983) 413 [INSPIRE].

[33] E. Cremmer et al., Vector Multiplets Coupled to $N=2$ Supergravity: SuperHiggs Effect, Flat Potentials and Geometric Structure, Nucl. Phys. B 250 (1985) 385 [inSPIRE].

[34] D.Z. Freedman, P. van Nieuwenhuizen and S. Ferrara, Progress Toward a Theory of Supergravity, Phys. Rev. D 13 (1976) 3214 [InSPIRE].

[35] S. Deser and B. Zumino, Consistent Supergravity, Phys. Lett. B 62 (1976) 335 [InSPIRE].

[36] A. Van Proeyen, Tools for supersymmetry, Ann. U. Craiova Phys. 9 (1999) 1 [hep-th/9910030] [INSPIRE].

[37] M. Henneaux, Consistent interactions between gauge fields: The cohomological approach, Contemp. Math. 219 (1998) 93 [hep-th/9712226] [INSPIRE].

[38] B. Julia, Effective Gauge Fields and Generalized Noether Theorem, in 4th Workshop on Current Problems in High-Energy Particle Theory Bad Honnef, Germany, June 2-4, 1980, pp. 295-313.

[39] G. Barnich, F. Brandt and M. Henneaux, Conserved currents and gauge invariance in Yang-Mills theory, Phys. Lett. B 346 (1995) 81 [hep-th/9411202] [INSPIRE]. 